\title{
Wireless Crack Monitoring by Stationary Phase Measurements from Coupled RFID Tags
}

\author{
Stefano Caizzone, Emidio DiGiampaolo, and Gaetano Marrocco
}

\begin{abstract}
The possibility to wirelessly monitor the state and the evolution of cracks is of increasing interest in emerging structural health monitoring systems. A simple and effective measurement method considers the placement of two passive radio frequency identification (RFID) antennas on top of the crack, so that the crack's evolution will produce a change of the inter-antenna coupling and in turn of the phase of the backscattered field. An ad-hoc design technique, based onto the coupled-modes physics, permits to maximize the sensor's sensitivity avoiding, or at least mitigating, the read range reduction during the evolution of the displacement that is instead typical of amplitude-oriented RFID displacement sensors. The proposed idea is demonstrated by numerical and experimental examples showing the possibility of sub-millimeter resolution with low-cost devices.
\end{abstract}

Index Terms-Array, deformation monitoring, mutual coupling, radio frequency identification (RFID), sensors, SHM, wireless communications.

\section{INTRODUCTION}

C IVIL, industrial and mechanical structures undergo fatigue processes, leading to "aging" and to limitation of their exploitable lifetime. It is, hence, critical to monitor the "health" of a structure in order for the operators (either civil engineers, maintenance technicians or others) to switch to a condition-based maintenance. An adequate monitoring system permits to observe the worsening of the structural conditions allowing a prompt repair work and avoiding more expensive and/or dangerous events [1].

Cracks are one of the most critical but also interesting phenomena for engineers, as they visually show the damage the structure is suffering from. Cracks are thin and usually jagged spaces opening in solid materials leading to fractures. Precise monitoring is of interest, meaning that desired resolutions are below $1 \mathrm{~mm}$ and ranges rarely go above one or few centimeters, as such crack dimensions would lead to structural collapse. It is currently possible to monitor crack evolution in quite a few different ways, namely, through ultrasonic testing, fiber optic sensors, metal foil strain gages, or via raw crackmeters (see

Manuscript received January 09, 2014; revised June 26, 2014; accepted August 16, 2014. Date of publication September 26, 2014; date of current version November 25, 2014.

S. Caizzone is with the Institute of Communications and Navigation, German Aerospace Center (DLR), Oberpfaffenhofen, Germany,, and also with the DISP, University of Rome, Tor Vergata, Italy (e-mail: stefano.caizzone@dlr.de).

E. DiGiampaolo is with the Dipartimento di Ingegneria Industriale e dell'Informazione e Economia, University of L'Aquila, Italy (e-mail: emidio.digiampaolo@univaq.it).

G. Marrocco is with the DISP, University of Rome, Tor Vergata, Italy (e-mail: marrocco@disp.uniroma2.it).

Color versions of one or more of the figures in this paper are available online at http://ieeexplore.iee.org.

Digital Object Identifier 10.1109/TAP.2014.2360553 for instance [2] for a review). The first ones are quite accurate measurement techniques, but they are often expensive and not easy to perform; strain gages are easier to install, but still require an extensive cabling, while with crackmeters, an operator has to manually record the dilatation of the crack by reading a mm-scale. It would be instead beneficial to have wireless, low-cost and passive sensors able to remotely provide the information about the state of the crack in a structure at a given time. Among the many possible technological options, sensors based on radiofrequency identification (RFID) [3] appear to be promising thanks to the maturity of commercial off-the-shelf (COTS) components, the virtually infinite lifetime of passive transponders and the overall low cost that would enable a widespread distribution over large areas, that is instead prevented by the high cost of active devices.

The recent attempts to use RFIDs to record and monitor the evolution of cracks in civil or mechanical structures may be categorized into two main families concerning deforming antennas and two-parts sensors. The first class of devices detects cracks by letting the tag antenna physically deform along with or be damaged by the crack enlargement. For instance an electrically conductive paint is used as part of the RFID tag in [4]: as the paint is deformed or broken due to the crack, its conductivity changes and the tag is mismatched, hence badly or even not communicating with the reader at all. In [5], the whole meander-line tag is deformed due to stress on the underlying object and, hence, it changes its backscattering properties. In [6], it is assumed that the crack will form a kind of slot on the patch antenna surface, changing the resonance behavior of the dominant modes of radiation. However, this solution needs a vector network analyzer (VNA) to record small frequency shifts and does not provide any clear ways of distinguishing between various sensors. In [7] a long transmission line is attached to an antenna and a time domain technique is used for data retrieval: if the crack damages the transmission line at a given position, the delay of the backscattered echo may be related to the position of such discontinuity. Also in this case, a wide frequency band and an expensive equipment is required and the identification of different sensors may be problematic. Finally, [8] shows how the strain occurring on a patch-type tag antenna due to stress forces, causes the substrate of the patch to deform, with variation in permittivity and, thus, also in resonant frequency. Good resolutions can be achieved, but accurate measurement of the frequency shift of the backscattered power is once more needed: that is not feasible with low-cost readers.

The second family of devices exploits a change of the mutual position between two parts of the radiating element of the tag. In [9] a two active-antennas system monitors the crack evolution by accurately detecting, through a complex measurement 
TABLE I

COMPARISON BetWeEn StATE-OF-THE-ART Wireless CRACK DETECTION Systems AND THE ONE PROPOSED IN THIS PAPER

\begin{tabular}{|c|c|c|}
\hline Method & Pros & Cons \\
\hline [4] & $\begin{array}{l}\text { Easy implementation; } \\
\text { IDentification through } \\
\text { RFID tech }\end{array}$ & $\begin{array}{c}\text { Threshold info only; No } \\
\text { crack evolution } \\
\text { monitoring possible }\end{array}$ \\
\hline [5] & $\begin{array}{l}\text { Good sensitivity; ID } \\
\text { through RFID tech }\end{array}$ & $\begin{array}{c}\text { Strong communication } \\
\text { degradation along with } \\
\text { sensing }\end{array}$ \\
\hline [6] & $\begin{array}{l}\text { Good sensitivity and 2D } \\
\text { crack detection }\end{array}$ & $\begin{array}{l}\text { Costly equipment needed; } \\
\text { difficult ID }\end{array}$ \\
\hline [7] & $\begin{array}{c}\text { Crack orientation } \\
\text { determination }\end{array}$ & $\begin{array}{l}\text { Costly equipment needed; } \\
\text { difficult ID }\end{array}$ \\
\hline [8] & $\begin{array}{l}\text { Good sensitivity; strain } \\
\text { and crack monitoring; ID } \\
\text { through RFID tech }\end{array}$ & Costly equipment needed \\
\hline [9] & Easy sensor structure & $\begin{array}{l}\text { Complicated read } \\
\text { procedure }\end{array}$ \\
\hline$[10]$ & $\begin{array}{l}\text { Good sensitivity and } \\
\text { setup independence }\end{array}$ & $\begin{array}{l}\text { Strong communication } \\
\text { degradation along with } \\
\text { sensing }\end{array}$ \\
\hline $\begin{array}{l}\text { This } \\
\text { paper }\end{array}$ & $\begin{array}{l}\text { Good sensitivity and } \\
\text { stable communication }\end{array}$ & Fixed setup needed \\
\hline
\end{tabular}

procedure, the angular shift of a null of the radiation pattern produced by the couplet of tags. A tag composed of two mutually sliding parts is described in [10]: as the crack enlarges, one part slides over the other with the purpose of detuning the antenna. Remarkable resolutions are shown at the expenses of a degraded communication capability due to the antenna detuning along with displacement.

Table I shows briefly advantages and disadvantages of the presented state of the art solutions.

The subset of above systems based on battery-less (passive) transponders achieves sensing capability throughout measurement of the amplitude of the signal backscattered by the tag. The present paper introduces instead a completely different approach, since it exploits the phase of the backscattered signal as carrier of the sensing information, while minimizing the degradation of communication performance by means of an ad-hoc design procedure. Working with phase turns out to be attractive, especially if using a COTS reader to collect measurements, thanks to:

i) the large dynamic range of phase measurements (between $0^{\circ}$ and $180^{\circ}$ );

ii) the possibility, as shown later on, to overcome or at least mitigate, the degradation of the communication performances typical of amplitude-oriented sensing method (see [3] and [11] for a detailed review).

The phase change is achieved by letting the crack's displacement modify the electromagnetic coupling among two facing tags under couplet-mode radiation. Modelling will be shown in Section II and a design procedure will be discussed in Section III. The idea will be then demonstrated by means of numerical examples (Section IV) and experimental tests with fabricated prototypes (Section V).

\section{GeOmetrical Displacement AND RFID Phase}

On considering that a crack forms two different parts sliding away from each other, the proposed sensor comprises two facing

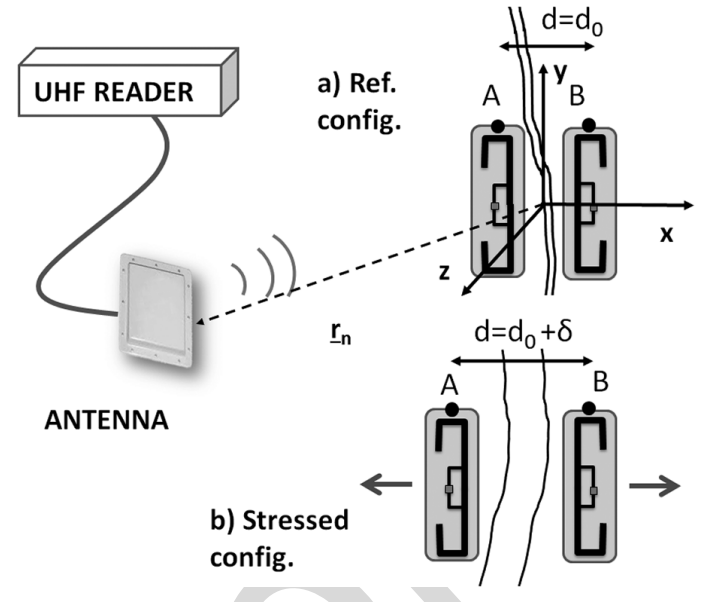

Fig. 1. System-level representation of the proposed RFID crack sensor, positioned on a wall and communicating with a remote RFID reader. a) Reference configuration, with the two tags on the two edges of the crack. b) Stressed configuration, with the two tags further apart from each other, due to mechanical stress enlargening the crack.

RFID tags (Fig. 1) as in [10] placed over the surface hosting the crack at opposite sides of it, for instance fixed by means of glue or screws. An RFID reader illuminates the scene from such a distance to be able to turn on the tags and collect their responses. Denoting with A and B two reference points of those tags, the inter-tag distance will be hereafter denoted as $d=\overline{A B}$, while the initial distance (before the stress is applied) is $d_{0}=$ $\overline{A B}(t=0)$. It is assumed for simplicity that the couplet of tags is subjected to a 1-D stress parallel to the $\mathrm{AB}$ direction.

The evolution of the crack will produce a displacement $\delta$ between points A, B so that $d=d_{0}+\delta$. Such displacement $\delta$ has to be detected by processing the RFID signals collected by the reader.

Being the two tags in close proximity, their electromagnetic response to the reader will be strongly dependent on the change of their mutual position $\mathrm{AB}$ so that the unknown instantaneous displacement can be estimated by processing the response of the tag systems at different times. In particular, the here considered tags' responses are the phases of the backscattered signals which, as shown in the next paragraph, can be formally related to the geometrical and electrical parameters of the coupled tags. Assuming a fixed measurement setup and that the reader-tag distance is much larger than the inter-tag distance $(r \gg d)$; hence, the change in the RFID responses at different times can be considered as only related to the unknown displacement $\delta$.

\section{A. Phase Response of a Couplet of RFID Tags}

The electromagnetic modulated backscattering of a couplet of tags can be described by means of a multiport network as in [12] (where the theoretical background and details can be found). The signal $V_{R,(n)}^{O N / O F F}$ collected at the reader's terminals due to the antenna mode of tag $n$ in a symmetrical couplet of identical tags is easily found to be

$$
V_{R,(n)}^{\frac{O N}{O F F}}[d]=\frac{Z_{n n}+Z_{m m}-2 Z_{m n}+Z_{C, m}+Z_{C, n}^{\frac{O N}{O F F}}}{\left(Z_{n n}+Z_{C, n}^{\frac{O N}{O F}}\right)\left(Z_{m m}+Z_{C, m}\right)-Z_{m n}^{2}} f\left(r_{n}\right)
$$


where $Z_{m n}[d]\{m, n=1,2\}$ are the elements of the impedance matrix of the two-port network referred to the terminals of the two tags and dependent on the inter-tag distance, $Z_{C, n}^{O N / O F F}$ and $Z_{C . m}$ are the modulating and the harvesting-mode impedances of the two chips, respectively. $f\left(r_{n}\right)$ is a complex function accounting for the parameters of the antennas and for the reader's parameters as well as for the propagation distance $r_{n}$ between the $n$th tag and the reader antennas

$$
f\left(r_{n}\right) \propto \frac{\lambda^{2}}{\pi^{2}} \sqrt{\frac{P_{i n} R_{R}^{r a d}}{2}} G_{R} R_{n}^{r a d} G_{n}\left(\underline{\hat{h}}_{n} \cdot \underline{\hat{h}}_{R}\right)^{2} \frac{e^{-j 2 k r_{n}}}{4 r_{n}^{2}}
$$

where $\lambda$ is the wavelength, $P_{i n}$ is the power supplied by the reader, $R_{R}^{r a d}$ and $R_{n}^{r a d}[d]$ are, respectively, the radiation resistance of the reader and of the $n$th tag antenna, $G_{R}$ is the gain of the reader antenna, $G_{n}[d]$ the gain of the $n$th tag (when the other tag is in open circuit condition), $\underline{\hat{h}}_{R}$ and $\underline{\hat{h}}_{n}[d]$ are respectively the polarization unitary vector of the reader and of the $n$th tag antenna. Since the displacements of interest (millimeter and sub-millimeter scale) are much smaller than the reader-to-tag distance, it is here and in the following assumed that the effective heights of the couplet will undergo negligible variations with mutual distance.

The phase of the signal backscattered from the $n$th modulating tag and retrieved by a commercial reader can be extracted (as for instance reported in [13]) by the differential received signal among the two modulating impedance states

$$
\varphi_{n}[d]=\arg \left\{V_{R,(n)}^{O F F}[d]-V_{R,(n)}^{O N}[d]\right\} .
$$

Introducing (1) in (3) and denoting with $Z_{S}=Z_{11}=Z_{22}$ (self impedance) and $Z_{M}=Z_{21}=Z_{12}$ (mutual impedance), and considering a reference modulation scheme [14] having $Z_{C . n}^{O N}=$ $Z_{C, m}=Z_{C}$ and $Z_{C, n}^{O F F} \rightarrow \infty$; hence, (3) becomes

$\varphi_{n}[d]=\arg \left\{-\frac{Z_{S}[d]-Z_{M}[d]+Z_{C}}{\left(Z_{S}[d]+Z_{C}\right)\left(Z_{S}[d]+Z_{M}[d]+Z_{C}\right)}\right\}+\varphi_{0}$

where the baseline phase $\varphi_{0}=\arg \left\{f\left(r_{n}\right)\right\}$ is independent from the impedances and will not change if the relative distance between the reader and the tag remains stable in successive measurements (fixed measurement setup assumption), under the simplifying hypothesis previously made.

The unknown displacement $\delta$ between A, B points, and, hence, the evolution of the crack, can be hence formally estimated by inverting the calibration curve obtained as phase difference at a given time with respect to the initial reference condition

$$
\delta \leftrightarrow \Delta \varphi_{n}=\varphi_{n}[d]-\varphi_{n}\left[d_{0}\right] .
$$

\section{Antenna Design Problem}

Equation (5) gives a sensing metric to be considered in the application of the two-tags system whose performance can be quantified in terms of sensitivity $S=\partial \varphi / \partial \delta$. Anyway, since it has been demonstrated [11] that the features of a tag-as-a-sensor are generally obtained at the expenses of the degradation of the electromagnetic performance (the read distance), the design of the considered displacement sensor has also to cope with constraints over the active input impedance [12], [20] of the tags, which, if the reader illuminates the tags from boresight direction, can be written as

$$
Z_{i n, n}=Z_{S}+Z_{M} .
$$

The maximum power delivered to the chip, and, therefore, the maximum read distance, is obtained under Hermite matching condition $Z_{i n, n}=Z_{S}+Z_{M}=Z_{c h i p}^{*}$. The active input impedance can, hence, be assumed as the metric for the communication performance of the tag, since it is essential to guarantee maximum power delivered to the chip all along the sensing process.

It is worth noticing that being the self and mutual impedances dependent on the distance between the two tags, the active input impedance, and accordingly, the read distance of the two tags, will be sensitive to the displacement $\delta$. Thus, the goal of the antenna design is to achieve the maximum phase variation and the minimum change of the input impedance all along the evolution of the crack. An idea about how to master these constraints comes from a deeper understanding of the electromagnetic coupling of tags in case of very small inter-antenna distance.

\section{A. Extremely Coupled Antennas}

For two very close antennas, as in the case of the proposed two-tags displacement sensor, the coupled-modes theory [15] shows the onset of two families of current distributions, denoted as even and odd modes, according to the directions assumed by the induced currents over the antennas. The excitation of one or both the modes depends on the feeding modality, including the direction and polarization of the incoming field in case of receiving antennas. It is possible to define active input impedances for the even and odd modes as a function of the impedance matrix

$$
\begin{aligned}
& Z_{e}(d)=Z_{S}(d)+Z_{M}(d) \\
& Z_{o}(d)=Z_{S}(d)-Z_{M}(d) .
\end{aligned}
$$

More specifically, the two-tags RFID sensor, by the effect of the very close proximity, can be assumed to be excited by equalphase and equal-amplitude currents induced by the impinging field and, hence, the sensor will sustain only the even mode radiation.

As a matter of fact, the active input impedance of the 2-ports RFID grid, as defined in (6), corresponds to the input impedance (7) of the even-mode and, therefore, (4) and (6) can be rewritten (having omitted for notation simplicity the dependance on $d$ ) by introducing (7) and (8) as

$$
\varphi_{1}=\arg \left\{-\frac{\left(Z_{o}+Z_{C}\right)}{\left[\frac{1}{2}\left(Z_{e}+Z_{o}\right)+Z_{C}\right]\left(Z_{e}+Z_{C}\right)}\right\}+\varphi_{0}
$$

$$
Z_{i n, n}=Z_{e}
$$

As a consequence, the degradation of communication performance versus changes in the mutual distance of tags will be minimized if the even mode does not vary strongly along with the sensing process. The phase, instead, depends in a nonstraightforward manner on both even and odd-mode impedance and it is, hence, expected to exhibit substantial changes when either $Z_{e}$ or $Z_{o}$ have strong variations with mutual distance. In other 


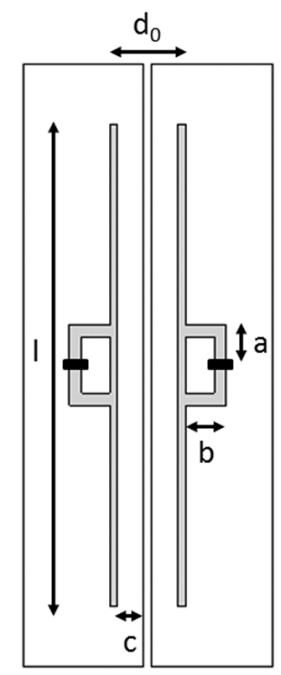

Fig. 2. Closely coupled T-match dipoles over a $150 \times 30 \times 0.8 \mathrm{~mm}$ FR- 4 slab. Size in [mm]: $a=10, b=12, c=6.5, l=120$, while $d$ is considered variable.
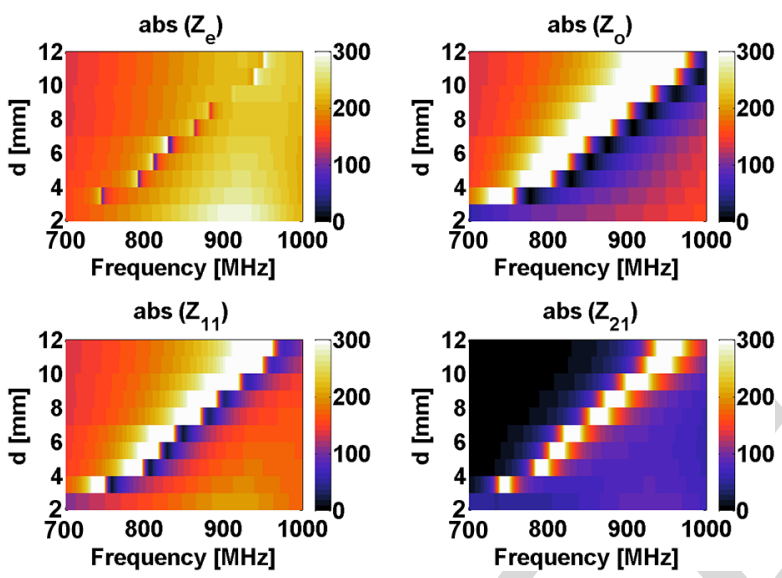

Fig. 3. Simulated even and odd mode (top) and self and mutual (bottom) impedances (absolute values) versus frequency and displacement for the two strongly-coupled T-match dipoles of Fig. 2.

words, conditions have to be found such that the even mode impedance is stable over inter-tag distance evolution, while the odd mode changes substantially.

The above concepts can be made explicit by the help of an example concerning two closely coupled T-match dipoles (Fig. 2) laying onto an FR-4 substrate.

Fig. 3 shows the absolute value of self, mutual, even and odd impedances with respect to inter-antenna distance and frequency, as obtained by numerical simulation through the electromagnetic solver CST Microwave Studio [18]. It is possible to clearly observe that self, mutual and odd impedances experience strong variations over frequency and over inter-antenna distance, while the even mode impedance is considerably more stable.

On recalling that $Z_{e}=Z_{i n, n}$, the combinations of $\{d, f\}$ around the main diagonal of the diagrams in Fig. 3 are particularly interesting since the input impedance of the tags remains quite stable for various mutual distances, while at the same time self and mutual impedances, as well as the odd mode impedance, change rapidly.
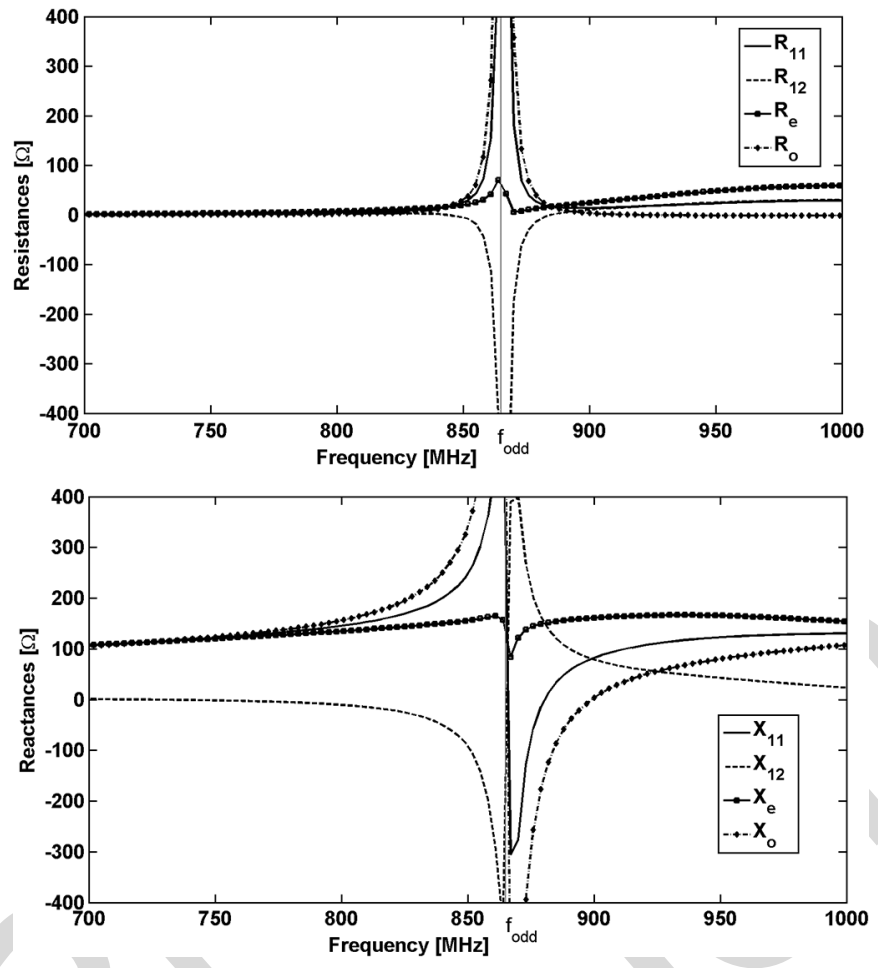

Fig. 4. Self and mutual resistance (top) and reactance (bottom) for the simulated couplet of the T-match tags of Fig. 2 at a mutual distance of $d_{0}=7 \mathrm{~mm}$ : the onset of the even and odd modes is clearly visible.

Forcing the tags to operate in the high-impedance band of Fig. 3 will, therefore, allow large phase variations with moderate degradation of impedance matching.

More in details, Fig. 4 shows a cut of previous impedance maps for a fixed inter-antenna distance $d=7 \mathrm{~mm}$ : the frequency region where the interesting phenomena occur corresponds to the parallel resonance of the odd mode input impedance and can be easily identified. These properties are of structural kind and hold also for different antenna layouts.

\section{B. Design Procedure}

The structural properties of the two closely coupled tags can be used to set up a design procedure that aims at forcing the two tags to operate in the high sensitive impedance region of Fig. 3. Let $\boldsymbol{\alpha}=\left\{\alpha_{1}, \alpha_{2}, . ., \alpha_{M}\right\}$ denote the set of geometrical and/or electrical parameters of the tags to be optimized, formally including also the initial inter-tag distance. However, for the sake of simplicity, and to speed-up the optimization procedure, a fixed reference distance $d=d_{0}$ is hereafter assumed. For instance, $d_{0}$ could be the smallest inter-tag distance that is compatible with the mechanical alignment of real antennas, including the substrates and the fixing method. The useful span of displacement detectable by the sensor will be verified a-posteriori at the end of the optimization procedure. As shown later on in the example section, the definitive initial inter-tag spacing for application on the crack will be then chosen according to the expected expanding or compressing behavior of the crack or, more in general, the initial distance could be allocated in the middle of the useful displacement range.

Parameters $\alpha$ have to be selected so that: 
i) the odd-mode anti-resonant frequency be tuned to the RFID frequency, i.e. $f_{\text {res,ODD }} \rightarrow f_{R F I D}$ to achieve enough sensitivity and negligible variation of the active input impedance and, hence, of the tags' range during the surface deformation process;

ii) the active input impedance be subjected to Hermite matching e.g. $Z_{i n, n}[d]=Z_{e}[d] \approx Z_{C}^{*}$ to enable the optimal delivery of scavenged power to the tags' microchip.

The above constraints are included into a penalty function $P[\boldsymbol{\alpha}]$

$$
P[\boldsymbol{\alpha}]=w_{1} \frac{\left|Z_{i n, n}[\boldsymbol{\alpha}]-Z_{c}^{*}\right|}{\left|Z_{c}^{*}\right|}+w_{2} \frac{\left|f_{\text {res }, O D D}[\boldsymbol{\alpha}]-f_{R F I D}\right|}{\left|f_{R F I D}\right|}
$$

to be minimized by means of any optimization tool. The weighting coefficients $w_{1}$ and $w_{2}=1-w_{1}$ may be freely chosen to emphasize sensing or communication performance.

For each guess of antenna parameters $\boldsymbol{\alpha}^{(k)}$, the anti-resonance frequency $f_{\text {res }, O D D}\left[\boldsymbol{\alpha}^{(k)}\right]$ of the odd-mode impedance (8) is detected by a zero-seeking procedure and compared with the RFID frequency. The impedance mismatch is estimated from (6) for $d=d_{0}$ and compared with the ideal Hermite matching condition, hence contributing to the evaluation of the penalty function.

Once the optimal antenna parameters have been determined through optimization, a further numerical electromagnetic simulation is performed, varying the inter-antenna distance $d$ around the reference distance $d_{0}$, in order to calculate the spacing-dependent two-port impedance matrix and to accordingly estimate the variation of the phase and of the realized gain all along the displacement process. A design example is given in the next section.

\section{NumERICAL EXAMPLES}

The application of the above design procedure is now discussed in detail by means of the same T-Match dipoles layout as in Fig. 2. The tags are assumed to operate in the European UHF band 865.6-867.6 MHz and to be connected to RFID microchips having input impedance $Z_{c, 1}=Z_{c, 2}=Z_{c}=15-$ $j 135 \Omega$ at $f_{R F I D}=867 \mathrm{MHz}$ (resembling that of NXP G2XM chip). The geometrical parameters of the two-tags sensor that are available to the optimization procedure are the T-match's form factor $\{a, b\}$ and the dipole length $l$ so that $\boldsymbol{\alpha}=\{a, b, l\}$. In particular, $\{a, b\}$ are known to mostly affect the impedance matching while $l$ impacts on the resonant frequency. The reference inter-dipole distance is fixed to $d_{0}=7 \mathrm{~mm}$ and the tag responses will be evaluated for $|\delta| \leq 5 \mathrm{~mm}$. In these conditions, the two tags are partially overlapping because of the dimensions of the tags shown in Fig. 2 and one tag slides over the other.

Fig. 5 shows the penalty function computed as described above with respect to parameters $b / a$ and $l$ and for the choice of the weights $w_{1}=0.3, w_{2}=0.7$, i.e. giving slightly more attention to the device sensitivity than to the communication capability. The penalty function gets minimized in the surrounding of $\{l, a, b\}=\{120,10,12\} \mathrm{mm}$. In this condition, we obtain $Z_{\text {in }}\left(f_{\text {RFID }}\right)=8+j 145 \Omega$ (to be compared with the conjugate of the microchip impedance) and $f_{\text {res }, O D D}=862 \mathrm{MHz}$ (to be compared with the European UHF RFID frequency).

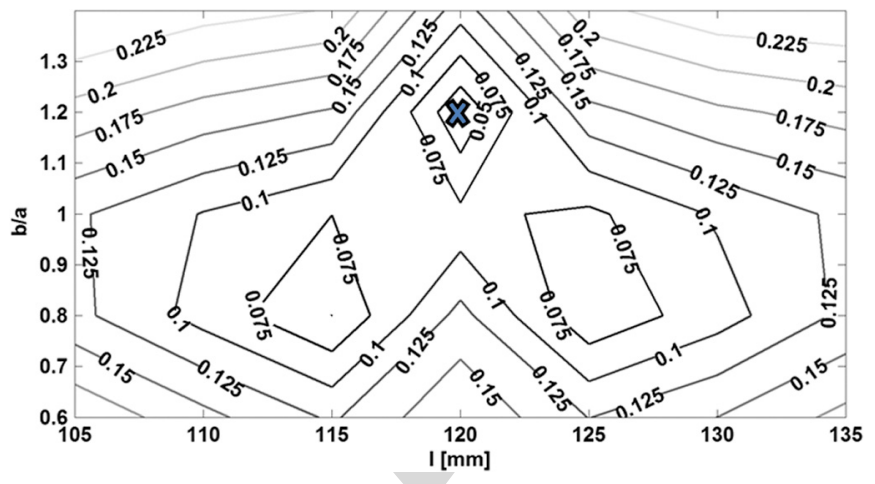

Fig. 5. Contours of the penalty function $P[l, b / a]$ for the two-dipoles sensor at $f_{R F I D}=867 \mathrm{MHz}$ (having chosen $w_{1}=0.3, w_{2}=0.7$ ), with respect to the dipole length and the T-match aspect ratio. The optimal solution is marked by a "X".

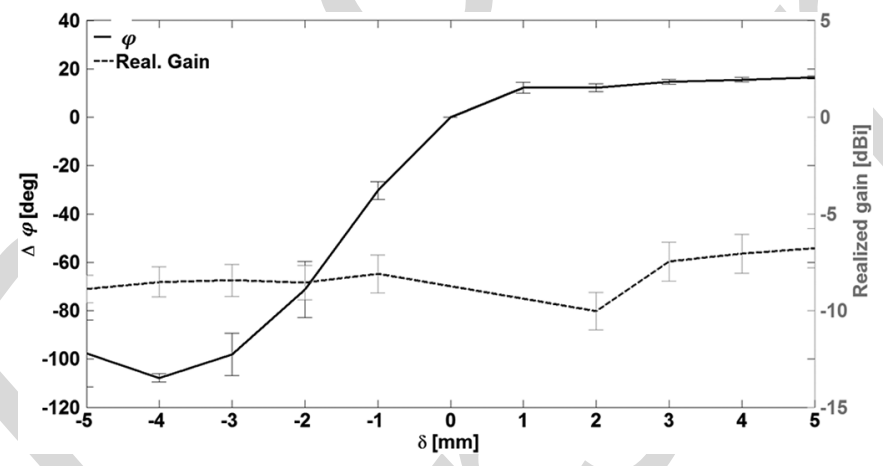

Fig. 6. Simulated phase calibration-curve (straight line) at $f=867 \mathrm{MHz}$, and realized gain (dotted line) versus the displacement $\delta$ among dipoles with respect to the reference configuration $d_{0}=7 \mathrm{~mm}$, so that $\overline{A B}=d_{0}+\delta$. Error bars indicate the sensitivity of the tag's response to $\pm 10 \%$ uncertainty in the microchip impedance w.r.t. the nominal value.

Finally, the calibration curve relating the relative phase $\triangle \varphi(\delta)$ computed as in (5) is represented in Fig. 6 together with the realized gain of the two tags-sensor, that can be considered an indirect measure of the impedance matching of the tags, easily retrieved by measurement throughout the turn-on method, as shown later in the experimental section. ${ }^{1}$

A monotonic trend is visible for the phase versus the intertags displacement with a nearly linear profile for $-4 \mathrm{~mm} \leq$ $\delta \leq 1 \mathrm{~mm}$ for an overall phase dynamic range of $120^{\circ}$. The estimated sensitivity is $S(\delta)=\partial \varphi / \partial \delta \sim 20[\mathrm{deg} / \mathrm{mm}]$. The realized gain is instead quite constant throughout the useful displacement range and is such to provide a maximum read distance of $3 \mathrm{~m}$ (for $\mathrm{EIRP}=3.2 \mathrm{~W}$ ) in free space condition.

The error bars in the sensor's response are related to a possible $\pm 10 \%$ uncertainty over the true input impedance of the RFID chip which is subjected to changes with respect to the nominal value provided by the data-sheet, depending on the fixing modality and on the incident power level. Although not negligible, such uncertainty does not invalidate the overall behavior of both phase and realized gain versus displacement and can afterwards be removed through calibration of the sensor.

\footnotetext{
${ }^{1}$ The realized gain of the two tags' sensor, which is directly proportional to the read distance, is defined [12] as $\widetilde{G_{n}}=G_{n}\left(4 R_{C} R_{S}^{r a d} /\left|Z_{i n, n}+Z_{C}\right|^{2}\right) \chi_{n}$, where $\chi_{n}$ is the polarization mismatch between reader and tag, $R_{C}=\operatorname{Re}\left[Z_{c}\right]$ and $R_{S}^{\text {rad }}$ is the radiation resistance of each tag antenna in case the other is in open circuit condition.
} 


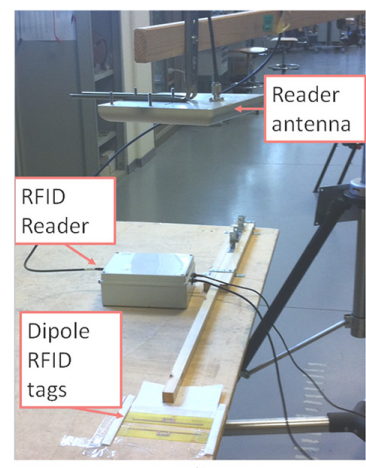

a)

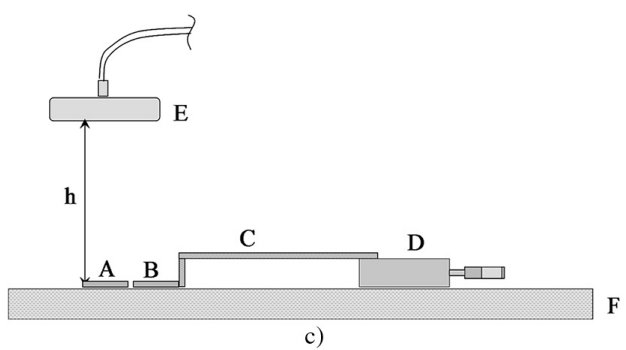

Fig. 7. a) Measurement setup, with two T-match tags over FR-4 placed on a wooden desk in front of the reader's antenna: the sliding tag translates by means of the linear translation stage guide. b) Zoom on the manufactured T-match tags. c) Detailed scheme of the set-up: A fixed tag; B sliding tag; C wooden rod; D linear translation stage guide; E reader's antenna; F wooden desk.

\section{EXPERIMENTATION}

The T-match dipole couplet of the previous Section has been manufactured using a drilling and milling machine for PCB prototyping. Antennas and T-match are carved out of a single-face FR-4 board $0.8 \mathrm{~mm}$ thick; NXP-G2XM microchips are soldered at the antenna terminals [Fig. 7(b)]. The two tags are closely deployed on a wooden desk so that one of the tags is fixed on the desk (fixed tag) while the other (sliding tag) shifts orthogonally (to the longer side) by means of a linear translation stage guide with resolution $\Delta \delta=10^{-2} \mathrm{~mm}$ [Fig. 7(a)]. The reader's antenna, a circularly polarized patch with $6 \mathrm{dBi}$ gain, is standing above the tags at distance $h=0.7 \mathrm{~m}$ and is connected to a ThingMagic M6e reader [19] set for fixed frequency operation and having disabled the frequency hopping. The reference inter-tag distance was fixed to $d_{0}=7 \mathrm{~mm}$, as in the optimization task. The sliding tag was progressively moved in the range $d_{0}-5 \mathrm{~mm}<d<d_{0}+5 \mathrm{~mm}$ by steps of 0.5 $\mathrm{mm}$ for an overall displacement of $10 \mathrm{~mm}$. RFID interrogations are performed at each steps: measurements consist in collecting the phase of the received backscattered signals and the turn-on power, which will be used to calculate the realized gain, as in [17].

Two measurement sessions have been performed for co-axial orientation between reader and tags (boresight measurement) and for off the axis placement (off-boresight measurement) with the aim to investigate the impact of imperfect alignments.

\section{A. Measurement Along the Boresight}

In a first experiment, the reader's boresight is aligned to the two-tags' normal axis so that the hypothesis in Section II are fully matched. The variation of the phase of the two tags recorded at the reader versus the distance between the two tags

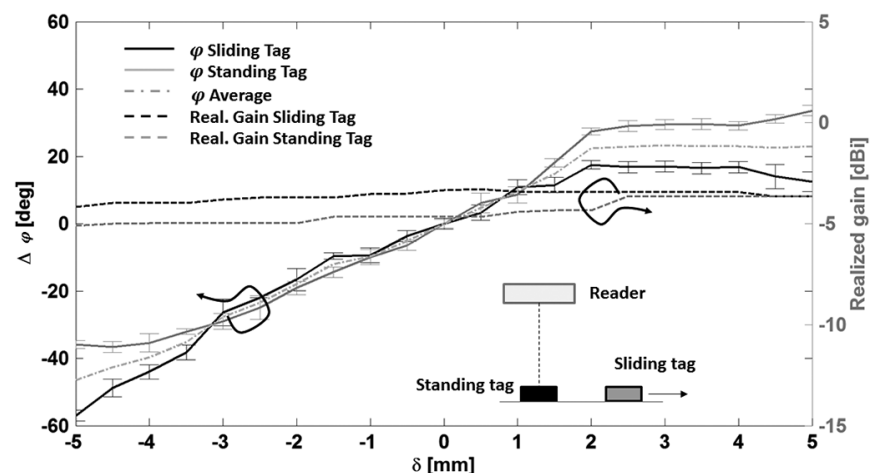

Fig. 8. Measured phase variation and realized gain (at $867 \mathrm{MHz}$ ), for the fixed and sliding tags, versus displacement $\delta$ among tags with respect to reference inter antenna distance $d_{0}=7 \mathrm{~mm}$ in case of along boresight illumination. Bars indicate the standard deviation in repeated measurements.

with increasing steps of $\Delta \delta=0.5 \mathrm{~mm}$ is shown in Fig. 8, together with the corresponding realized gain. Vertical bars in the diagram indicate the standard deviation in repeated measurements which resulted to be less than $3^{\circ}$. The useful part of the dynamic range of the sensor, wherein the profile is mostly linear, is $-4 \mathrm{~mm} \leq \delta \leq 2 \mathrm{~mm}$. It is worth specifying that, having assumed an arbitrarily reference initial inter-antenna spacing $d_{0}=7 \mathrm{~mm}$, the absolute spacing that can be sensed by the systems will be $3 \mathrm{~mm}<d=d_{0}+\delta<9 \mathrm{~mm}$. Hence, if, for instance, an expanding crack is expected, the full available $6 \mathrm{~mm}$ sensing range can be exploited by fixing the two antenna at an initial distance $d^{\prime}=3 \mathrm{~mm}$. The experimented sensitivity is $S_{\text {meas }}(\delta) \sim 10 \mathrm{deg} / \mathrm{mm}$ in the range $\delta=\{-4 ; 2\} \mathrm{mm}$ to be compared with the theoretical value $S_{\text {theor }}(\delta) \sim 20 \mathrm{deg} / \mathrm{mm}$. Differences are mostly due to the presence of the wooden desk set-up, on which tags are placed and that was instead not considered in the optimization.

Finally, it is worth noticing that the phase profiles of the sliding and standing tags are quite similar in the useful linear region, while some asymmetries are apparent elsewhere, maybe due to the partial overlapping of the FR-4 supports of the two tags and to the potential difference between the two microchip RF impedances, as discussed in Fig. 6. It is, hence, suggested to perform a calibration task (numerical and/or experimental), at least for the specific class of applications, to get rid of possible material-dependent uncertainty, before real-life use.

\section{B. Off Boresight Measurement}

A second set of experiments (Fig. 10) considers shifts and rotations (Fig. 9) of the reader's antenna w.r.t. the boresight alignment, so that the two-tags sensor is illuminated from different positions $\{\theta, \phi, r\}$. These experiments permit to evaluate the immunity of the sensor's response from the particular choice of mutual reader-tag alignment (that, however, is considered fixed during each measurement session of the inter-tag displacement).

Fig. 10 shows the results for a moderate deviation from the boresight illumination and for $10 \mathrm{~cm}$ increase in the reader-tag distance $\left(\{\theta, \phi, r\}=\left\{25^{\circ}, 90^{\circ}, 80 \mathrm{~cm}\right\}\right)$. The obtained displacement-phase profile fully compares with that of the reference boresight case. 


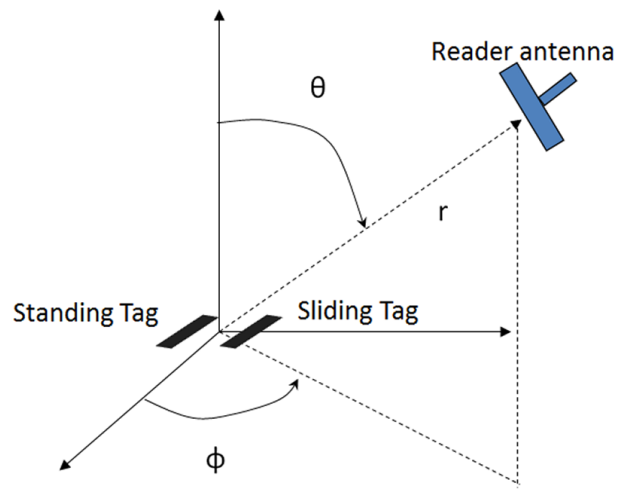

Fig. 9. Geometrical setup used to test the sensor's response for different readertags positions.

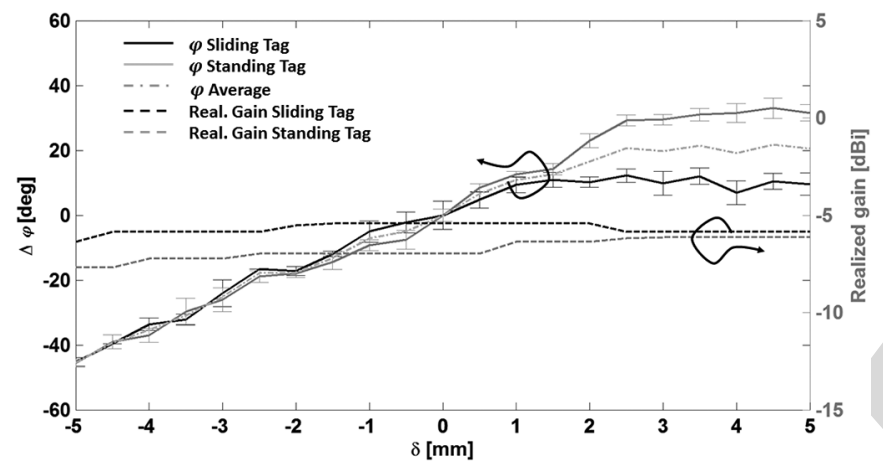

Fig. 10. Measured phase variation and realized gain (at $867 \mathrm{MHz}$ ), for the fixed and sliding tags, versus displacement $\delta$ among tags with respect to reference inter-antenna distance $d_{0}=7 \mathrm{~mm}$ in case of off-boresight illumination $\theta, \phi, r=\left\{25^{\circ}, 90^{\circ}, 80 \mathrm{~cm}\right\}$.

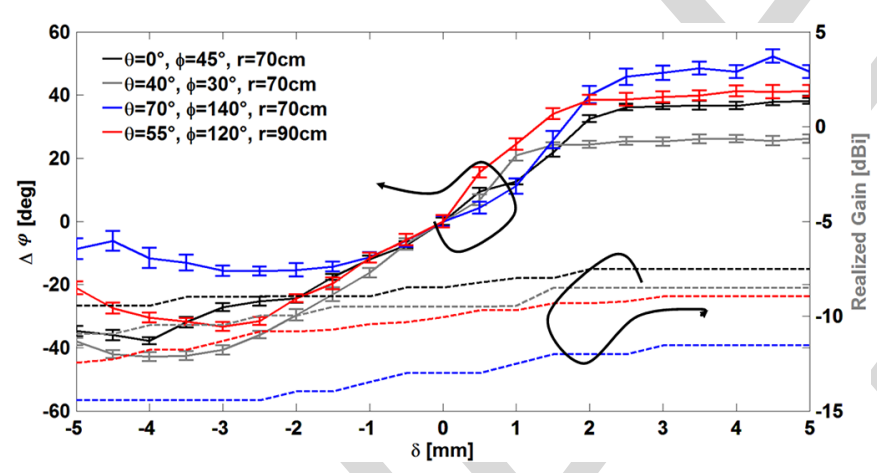

Fig. 11. Measured phase variation and realized gain (at $867 \mathrm{MHz}$ ) versus displacement $\delta$ among tags with respect to reference inter-antenna distance $d_{0}=$ $7 \mathrm{~mm}$ in case of several off-boresight illumination.

Results for more severe grazing illuminations $\left(40^{\circ}<\theta<\right.$ $\left.70^{\circ}\right)$ are summarized in Fig. 11 for the fixed tag, at a distance up to $r=90 \mathrm{~cm}$ from the reader.

On comparing the response profiles to the one obtained for boresight illumination, it is possible to appreciate a substantial stability in the central part of the linear region (around $-3 \mathrm{~mm}<\delta<2 \mathrm{~mm}$ ) except for the very low elevation angles $\left(\theta=70^{\circ}\right)$, where the simplifying hypotheses of Section II are not fulfilled anymore.

It is worth concluding that, while the sensor's gain is quite strongly affected by the mutual orientation between devices, the reader-tag mutual position will not be instead a critical issue in

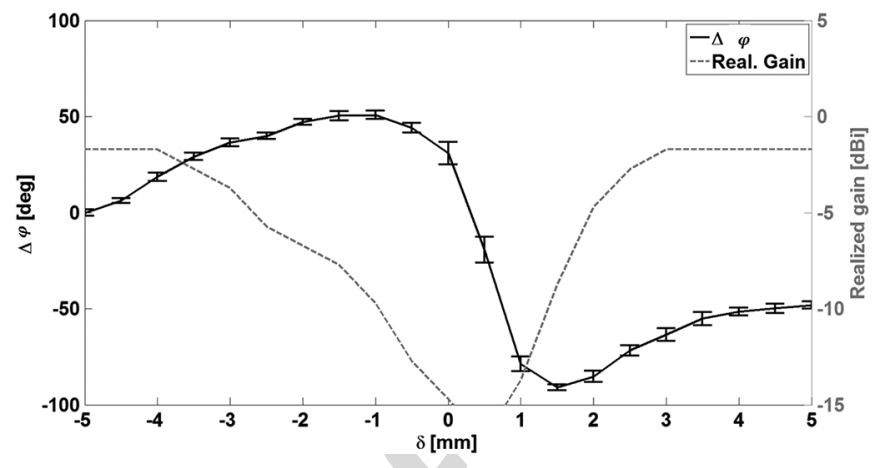

Fig. 12. Measured phase variation and realized gain (at $867 \mathrm{MHz}$ ) versus displacement $\delta$ among tags with respect to reference inter-antenna distance $d_{0}=$ $7 \mathrm{~mm}$ in case of only one tag with chip and the other in OC; boresight illumination.

real life phase measurements, provided that the elevation angles does not roughly exceed $45^{\circ}$. It is, however, advisable to perform calibration with the same (fixed) setup used in measurement to reduce uncertainties related to the random mutual orientation.

\section{A Single-Chip Case}

It is finally useful to analyze the sensing performance achievable when only a single chip is used, e.g. when the chip of the sliding antenna is replaced by an open circuit. The resulting device will, hence, work as a moving disturbing scatterer for the true RFID tag, as in the case of the sliding patch in [10]. The balancing effect of the mutual interaction between the tags will be lost, since, if $Z_{C, 2} \rightarrow \infty$, phase and active input impedance will approach the simplified expressions

$$
\begin{aligned}
\varphi_{1} & \rightarrow \arg \left(\left(\frac{1}{Z_{S}+Z_{C, 1}}\right)\right)+\varphi_{0} \\
Z_{i n, 1} & \rightarrow Z_{s} .
\end{aligned}
$$

Since the self impedance exhibits strong variations at $f_{R F I D}$ for inter-tag displacements around $d=7 \mathrm{~mm}$ (as in Fig. 4), both phase and input impedance are expected to vary strongly.

Fig. 12 shows the measured results from laboratory experiments $\left(\theta, \phi, r=\left\{0^{\circ}, 0^{\circ}, 100 \mathrm{~cm}\right\}\right)$ : a much higher sensitivity $S(\delta)=150 \mathrm{deg} / \mathrm{mm}$ is achieved but within a more limited range of displacement $\delta=[0 ; 1] \mathrm{mm}$, and at the expense of a stronger degradation in the link range, since the realized gain $\widetilde{G_{n}}$ attenuates by more than $10 \mathrm{~dB}$.

\section{CONCLUSION}

Relating the phase of the backscattering power emitted by two strongly coupled facing tags to the change of their mutual position has been demonstrated to be a promising procedure to detect sub-millimeter displacements without producing strong communication degradation that is instead typical of amplitude-oriented RFID sensors. On considering that a commercial low-cost reader typically exhibits a phase resolution of $3^{\circ}$, the results of experimentation show that the proposed couplet of tags already allows a displacement resolution as low as $0.3 \mathrm{~mm}$ over a span of up to $6 \mathrm{~mm}$. That resolution could be improved by further optimization and trade-off analysis (i.e. by giving more 
emphasis to sensitivity rather than communication in the design procedure), as well as by using more stable readers with great benefit to spatial resolution. For instance, a phase deviation of $1^{\circ}$ could enable a displacement resolution of $0.1 \mathrm{~mm}$ with the proof-of-concept manufactured prototype: such value comes near the one of some well-assessed active measurement devices. In case very short range interrogations are acceptable, even sharper resolutions could be moreover achieved by using single-chip configurations where the dummy tag works as a sliding scatterer.

The proposed technique employing phase to retrieve sensing information needs a fixed measurement setup, even if it revealed rather robust to the imperfect alignment between reader's antenna and tags. The stationary-measurement requirement, that looks not suitable to the use of hand-held readers, is instead fully compliant with laboratory environments as well as with external real life applications provided that at least the reader's antenna (and not necessarily the whole reader unit) remains fixed with respect to the surface to be observed at different times. In this sense, the proposed arrangement could be particular feasible in avionic framework wherein the interrogating antenna could be integrated inside the structure.

\section{REFERENCES}

[1] C. Boller and N. Meyendorf, "State of the art in SHM for aeronautics," presented at the Int. Symp. NDT Aerospace, 2008.

[2] P. C. Chang, A. Flatau, and S. C. Liu, "Review paper: Health monitoring of civil infrastructure," Struct. Health Monit., vol. 2, no. 3, pp. 257-267, Sep. 2003.

[3] C. Occhiuzzi, S. Caizzone, and G. Marrocco, "Passive UHF RFID antennas for sensing applications: Principles, methods and classifications," IEEE Antennas Propagat. Mag., to be published.

[4] K. Morita and K. Noguchi, "Crack detection methods for concrete and steel using radio frequency identification and electrically conductive materials and its applications," Proc. SPIE, 2008.

[5] C. Occhiuzzi, C. Paggi, and G. Marrocco, "Passive RFID strain-sensor based on meander line antennas," IEEE Trans. Antennas Propag., vol. 59, no. 12 , pp. $4836-4840$, Dec. 2011.

[6] B. S. Cook, A. Shamim, and M. M. Tentzeris, "Passive low-cost inkjetprinted smart skin sensor for structural health monitoring," IET Microw. Antennas Propag., vol. 6, no. 14, pp. 1536-1541, Nov. 2012.

[7] P. Kalansuriya, R. Bhattacharyya, S. Sarma, and N. Karmakar, "Towards chipless RFID-based sensing for pervasive surface crack detection," in Proc. IEEE Conf. RFID- Technologies and Applications, Nov. 2012, pp. 46-51.

[8] X. Yi, C. Cho, C.-H. Fang, J. Cooper, V. Lakafosis, R. Vyas, Y. Wang, R. T. Leon, and M. M. Tentzeris, "Wireless strain and crack sensing using a folded patch antenna," in Proc. Eur. Conf. Antennas and Propagation, Mar. 2012, pp. 1678-1681.

[9] V. Rizzoli, A. Costanzo, E. Montanari, and A. Benedetti, "A new wireless displacement sensor based on reverse design of microwave and millimeter-wave antenna array," IEEE Sensors J., vol. 9, no. 11, pp. 1557-1566, Nov. 2009.

[10] C. Paggi, C. Occhiuzzi, and G. Marrocco, "Sub-millimeter displacement sensing by passive UHF RFID antennas," IEEE Trans. Antennas Propag., vol. 62, no. 2, pp. 905-912, Feb. 2014.

[11] C. Occhiuzzi and G. Marrocco, "Constrained design of passive UHF RFID sensor antennas," IEEE Trans. Antennas Propag., vol. 61, no. 6, pp. 2972-2980, Jun. 2013.

[12] G. Marrocco, "RFID grids: Part I-Electromagnetic theory," IEEE Trans. Antennas Propag., vol. 59, no. 3, pp. 1019-1026, Mar. 2011.

[13] P. V. Nikitin, R. Martinez, S. Ramamurthy, H. Leland, G. Spiess, and K. V. S. Rao, "Phase based spatial identification of UHF RFID tags," in Proc. IEEE Int. Conf. RFID, Apr. 2010, pp. 102-109.

[14] F. Fuschini, C. Piersanti, F. Paolazzi, and G. Falciasecca, "Analytical approach to the backscattering from UHF-RFID transponder," IEEE Antennas Wireless Propag. Lett., vol. 7, pp. 33-35, Feb. 2008.
[15] H. A. Haus and W. Huang, "Coupled-mode theory," Proc. IEEE, vol. 79, no. 10, pp. 1505-1518, Oct. 1991.

[16] S. Manzari, S. Pettinari, and G. Marrocco, "Miniaturised wearable UHF-RFID tag with tuning capability," Electron. Lett., vol. 48, no. 21, pp. 1325-1326, Oct. 2012.

[17] G. Marrocco and F. Amato, "Self-sensing passive RFID: From theory to tag design and experimentation," presented at the Eur. Microwave Conf., Sep. 2009

[18] [Online]. Available: http://www.cst.com

[19] [Online]. Available: http://www.thingmagic.com/embedded-rfidreaders/mercury6e

[20] C. Balanis, Antenna Theory- Analysis and Design. Hoboken, NJ, USA: Wiley, p. 413.

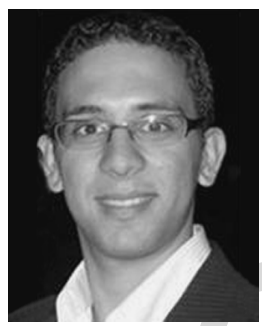

Stefano Caizzone received the M.Sc. degree in telecommunications engineering from the University of Rome Tor Vergata, Italy, in 2009. He is currently pursuing the Ph.D. degree.

His main research interests concern small antennas for RFIDs and navigation, antenna arrays, and grids with enhanced sensing capabilities. He is now with the Antenna Group, Institute of Communications and Navigation, German Aerospace Center (DLR), Wessling, Germany, where he is responsible for the development of innovative miniaturized antennas.

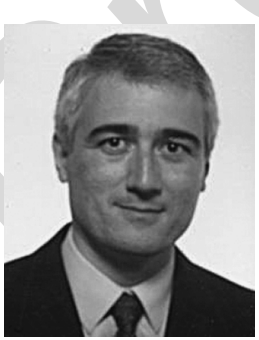

Emidio DiGiampaolo received the Laurea degree in electronic engineering and the Ph.D. degree in applied electromagnetics from the University of L'Aquila, Italy, in 1994 and 1998, respectively.

From 1998 to 2004, he was a postdoctoral researcher at the University of L'Aquila. In the spring of 2000, he was a visiting researcher at the European Space Research and Technology Centre (ESTEC), Noordwijk, The Netherlands. From 2005 to 2009, he was a researcher at the University of Rome Tor Vergata, Italy. Since 2010, he has been with the University of L'Aquila as an Assistant Professor. His research interests mainly concern numerical methods for modeling radio-wave propagation in complex environments, antennas, RFID, and radio localization.

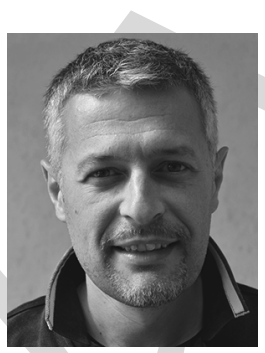

Gaetano Marrocco received the electronic engineering Laurea and the Ph.D. degree in applied electromagnetics from the University of L'Aquila, Italy, in 1994 and 1998, respectively.

In 1994, he was with the University of Illinois at Urbana-Champain as a postgraduate student In 1997, he joined the University of Roma Tor Vergata, Italy, as a researcher. In 1999, he was a visiting researcher at the Imperial College, London, U.K. Currently, he serves as Associate Professor of electromagnetics, University of Roma Tor Vergata, and he chairs the Pervasive Electromagnetics Lab. In 2014, he obtained the Full Professor qualification. In 2015, he will be a Guest Professor at the University of Paris-est Marne la Vallèe, France. His research is mainly focused on the application of radiofrequency identification (RFID) to medical and industrial diagnostics as well as to the modeling and design of distributed and miniaturized conformal antenna clusters over ships, micro and nano-satellites, and aircrafts within the framework of the European Space Agency, NATO, the Italian Space Agency, and the Italian Navy research projects. Moreover, he was active in the development of FDTD methods for microwave hyperthermia and in the modeling and design of pulsed arrays.

Prof. Marrocco serves as an Associate Editor of the IEEE ANTENNAS AND Wireless Propagation LetTers and the IEEE RFID VirTUAL JOURNAL and as Vice-Chair of the Italian delegation URSI Commission D: Electronics and Photonics. He was the Chair of Local Committee of EUCAP-2011, Rome, Italy, and TPC Chair of the 2012 IEEE-RFID TA, Nice, France. He is the cofounder and President of the University spin-off RADIO6ENSE that is active in the short-range electromagnetic sensing for industry, internet of things, and smart cities. 


\title{
Wireless Crack Monitoring by Stationary Phase Measurements from Coupled RFID Tags
}

\author{
Stefano Caizzone, Emidio DiGiampaolo, and Gaetano Marrocco
}

\begin{abstract}
The possibility to wirelessly monitor the state and the evolution of cracks is of increasing interest in emerging structural health monitoring systems. A simple and effective measurement method considers the placement of two passive radio frequency identification (RFID) antennas on top of the crack, so that the crack's evolution will produce a change of the inter-antenna coupling and in turn of the phase of the backscattered field. An ad-hoc design technique, based onto the coupled-modes physics, permits to maximize the sensor's sensitivity avoiding, or at least mitigating, the read range reduction during the evolution of the displacement that is instead typical of amplitude-oriented RFID displacement sensors. The proposed idea is demonstrated by numerical and experimental examples showing the possibility of sub-millimeter resolution with low-cost devices.
\end{abstract}

Index Terms - Array, deformation monitoring, mutual coupling, radio frequency identification (RFID), sensors, SHM, wireless communications.

\section{INTRODUCTION}

C IVIL, industrial and mechanical structures undergo fatigue processes, leading to "aging" and to limitation of their exploitable lifetime. It is, hence, critical to monitor the "health" of a structure in order for the operators (either civil engineers, maintenance technicians or others) to switch to a condition-based maintenance. An adequate monitoring system permits to observe the worsening of the structural conditions allowing a prompt repair work and avoiding more expensive and/or dangerous events [1].

Cracks are one of the most critical but also interesting phenomena for engineers, as they visually show the damage the structure is suffering from. Cracks are thin and usually jagged spaces opening in solid materials leading to fractures. Precise monitoring is of interest, meaning that desired resolutions are below $1 \mathrm{~mm}$ and ranges rarely go above one or few centimeters, as such crack dimensions would lead to structural collapse. It is currently possible to monitor crack evolution in quite a few different ways, namely, through ultrasonic testing, fiber optic sensors, metal foil strain gages, or via raw crackmeters (see

Manuscript received January 09, 2014; revised June 26, 2014; accepted August 16, 2014. Date of publication September 26, 2014; date of current version November 25, 2014.

S. Caizzone is with the Institute of Communications and Navigation, German Aerospace Center (DLR), Oberpfaffenhofen, Germany, and also with the DISP, University of Rome, Tor Vergata, Italy (e-mail: stefano.caizzone@dlr.de).

E. DiGiampaolo is with the Dipartimento di Ingegneria Industriale e dell'Informazione e Economia, University of L'Aquila, Italy (e-mail: emidio.digiampaolo@univaq.it).

G. Marrocco is with the DISP, University of Rome, Tor Vergata, Italy (e-mail: marrocco@disp.uniroma2.it).

Color versions of one or more of the figures in this paper are available online at http://ieeexplore.ieee.org.

Digital Object Identifier 10.1109/TAP.2014.2360553 for instance [2] for a review). The first ones are quite accurate measurement techniques, but they are often expensive and not easy to perform; strain gages are easier to install, but still require an extensive cabling, while with crackmeters, an operator has to manually record the dilatation of the crack by reading a mm-scale. It would be instead beneficial to have wireless, low-cost and passive sensors able to remotely provide the information about the state of the crack in a structure at a given time. Among the many possible technological options, sensors based on radiofrequency identification (RFID) [3] appear to be promising thanks to the maturity of commercial off-the-shelf (COTS) components, the virtually infinite lifetime of passive transponders and the overall low cost that would enable a widespread distribution over large areas, that is instead prevented by the high cost of active devices.

The recent attempts to use RFIDs to record and monitor the evolution of cracks in civil or mechanical structures may be categorized into two main families concerning deforming antennas and two-parts sensors. The first class of devices detects cracks by letting the tag antenna physically deform along with or be damaged by the crack enlargement. For instance an electrically conductive paint is used as part of the RFID tag in [4]: as the paint is deformed or broken due to the crack, its conductivity changes and the tag is mismatched, hence badly or even not communicating with the reader at all. In [5], the whole meander-line tag is deformed due to stress on the underlying object and, hence, it changes its backscattering properties. In [6], it is assumed that the crack will form a kind of slot on the patch antenna surface, changing the resonance behavior of the dominant modes of radiation. However, this solution needs a vector network analyzer (VNA) to record small frequency shifts and does not provide any clear ways of distinguishing between various sensors. In [7] a long transmission line is attached to an antenna and a time domain technique is used for data retrieval: if the crack damages the transmission line at a given position, the delay of the backscattered echo may be related to the position of such discontinuity. Also in this case, a wide frequency band and an expensive equipment is required and the identification of different sensors may be problematic. Finally, [8] shows how the strain occurring on a patch-type tag antenna due to stress forces, causes the substrate of the patch to deform, with variation in permittivity and, thus, also in resonant frequency. Good resolutions can be achieved, but accurate measurement of the frequency shift of the backscattered power is once more needed: that is not feasible with low-cost readers.

The second family of devices exploits a change of the mutual position between two parts of the radiating element of the tag. In [9] a two active-antennas system monitors the crack evolution by accurately detecting, through a complex measurement 
TABLE I

Comparison BetweEn STATE-OF-THE-ART Wireless CRACK Detection SYSTEMS AND THE ONE PROPOSED IN THIS PAPER

\begin{tabular}{|c|c|c|}
\hline Method & Pros & Cons \\
\hline \hline$[4]$ & $\begin{array}{c}\text { Easy implementation; } \\
\text { IDentification through } \\
\text { RFID tech }\end{array}$ & $\begin{array}{c}\text { Threshold info only; No } \\
\text { crack evolution } \\
\text { monitoring possible }\end{array}$ \\
\hline$[5]$ & $\begin{array}{c}\text { Good sensitivity; ID } \\
\text { through RFID tech } \\
\text { Strong communication } \\
\text { degradation along with } \\
\text { sensing }\end{array}$ \\
\hline$[6]$ & $\begin{array}{c}\text { Good sensitivity and 2D } \\
\text { crack detection }\end{array}$ & $\begin{array}{c}\text { Costly equipment needed; } \\
\text { difficult ID }\end{array}$ \\
\hline$[7]$ & $\begin{array}{c}\text { Crack orientation } \\
\text { determination }\end{array}$ & $\begin{array}{c}\text { Costly equipment needed; } \\
\text { difficult ID }\end{array}$ \\
\hline$[8]$ & $\begin{array}{c}\text { Good sensitivity; strain } \\
\text { and crack monitoring; ID } \\
\text { through RFID tech }\end{array}$ & $\begin{array}{c}\text { Costly equipment needed } \\
\text { Easy sensor structure }\end{array}$ \\
\hline$[9]$ & $\begin{array}{c}\text { Good sensitivity and } \\
\text { setup independence }\end{array}$ & $\begin{array}{c}\text { Strong communication } \\
\text { pegradation along with } \\
\text { sensing }\end{array}$ \\
\hline$[10]$ & $\begin{array}{c}\text { Good sensitivity and } \\
\text { stable communication }\end{array}$ & Fixed setup needed \\
\hline This & & \\
\hline
\end{tabular}

procedure, the angular shift of a null of the radiation pattern produced by the couplet of tags. A tag composed of two mutually sliding parts is described in [10]: as the crack enlarges, one part slides over the other with the purpose of detuning the antenna. Remarkable resolutions are shown at the expenses of a degraded communication capability due to the antenna detuning along with displacement.

Table I shows briefly advantages and disadvantages of the presented state of the art solutions.

The subset of above systems based on battery-less (passive) transponders achieves sensing capability throughout measurement of the amplitude of the signal backscattered by the tag. The present paper introduces instead a completely different approach, since it exploits the phase of the backscattered signal as carrier of the sensing information, while minimizing the degradation of communication performance by means of an ad-hoc design procedure. Working with phase turns out to be attractive, especially if using a COTS reader to collect measurements, thanks to:

i) the large dynamic range of phase measurements (between $0^{\circ}$ and $180^{\circ}$ );

ii) the possibility, as shown later on, to overcome or at least mitigate, the degradation of the communication performances typical of amplitude-oriented sensing method (see [3] and [11] for a detailed review).

The phase change is achieved by letting the crack's displacement modify the electromagnetic coupling among two facing tags under couplet-mode radiation. Modelling will be shown in Section II and a design procedure will be discussed in Section III. The idea will be then demonstrated by means of numerical examples (Section IV) and experimental tests with fabricated prototypes (Section V).

\section{Geometrical Displacement And RFID Phase}

On considering that a crack forms two different parts sliding away from each other, the proposed sensor comprises two facing

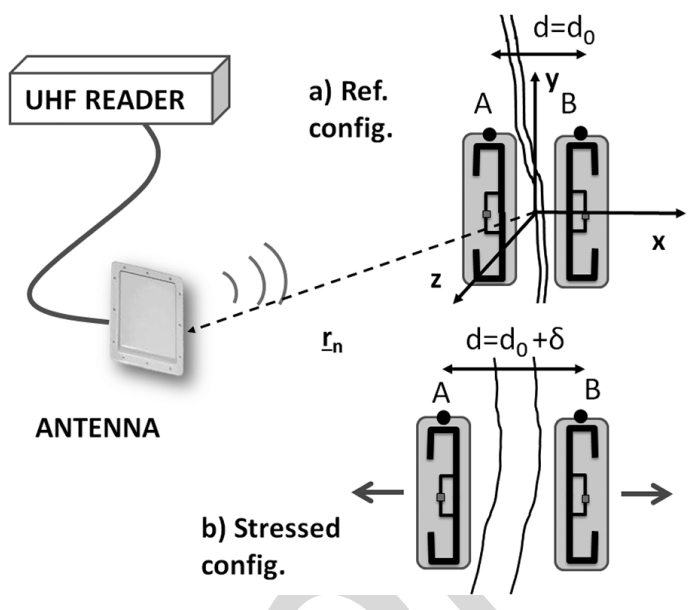

Fig. 1. System-level representation of the proposed RFID crack sensor, positioned on a wall and communicating with a remote RFID reader. a) Reference configuration, with the two tags on the two edges of the crack. b) Stressed configuration, with the two tags further apart from each other, due to mechanical stress enlargening the crack.

RFID tags (Fig. 1) as in [10] placed over the surface hosting the crack at opposite sides of it, for instance fixed by means of glue or screws. An RFID reader illuminates the scene from such a distance to be able to turn on the tags and collect their responses. Denoting with A and B two reference points of those tags, the inter-tag distance will be hereafter denoted as $d=\overline{A B}$, while the initial distance (before the stress is applied) is $d_{0}=$ $\overline{A B}(t=0)$. It is assumed for simplicity that the couplet of tags is subjected to a 1-D stress parallel to the $\mathrm{AB}$ direction.

The evolution of the crack will produce a displacement $\delta$ between points A, B so that $d=d_{0}+\delta$. Such displacement $\delta$ has to be detected by processing the RFID signals collected by the reader.

Being the two tags in close proximity, their electromagnetic response to the reader will be strongly dependent on the change of their mutual position $\mathrm{AB}$ so that the unknown instantaneous displacement can be estimated by processing the response of the tag systems at different times. In particular, the here considered tags' responses are the phases of the backscattered signals which, as shown in the next paragraph, can be formally related to the geometrical and electrical parameters of the coupled tags. Assuming a fixed measurement setup and that the reader-tag distance is much larger than the inter-tag distance $(r \gg d)$; hence, the change in the RFID responses at different times can be considered as only related to the unknown displacement $\delta$.

\section{A. Phase Response of a Couplet of RFID Tags}

The electromagnetic modulated backscattering of a couplet of tags can be described by means of a multiport network as in [12] (where the theoretical background and details can be found). The signal $V_{R,(n)}^{O N / O F F}$ collected at the reader's terminals due to the antenna mode of tag $n$ in a symmetrical couplet of identical tags is easily found to be

$$
V_{R,(n)}^{\frac{O N}{O F F}}[d]=\frac{Z_{n n}+Z_{m m}-2 Z_{m n}+Z_{C, m}+Z_{C, n}^{\frac{O N}{O F^{F}}}}{\left(Z_{n n}+Z_{C, n}^{\frac{O N}{O F}}\right)\left(Z_{m m}+Z_{C, m}\right)-Z_{m n}^{2}} f\left(r_{n}\right)
$$


where $Z_{m n}[d]\{m, n=1,2\}$ are the elements of the impedance matrix of the two-port network referred to the terminals of the two tags and dependent on the inter-tag distance, $Z_{C, n}^{O N / O F F}$ and $Z_{C, m}$ are the modulating and the harvesting-mode impedances of the two chips, respectively. $f\left(r_{n}\right)$ is a complex function accounting for the parameters of the antennas and for the reader's parameters as well as for the propagation distance $r_{n}$ between the $n$th tag and the reader antennas

$$
f\left(r_{n}\right) \propto \frac{\lambda^{2}}{\pi^{2}} \sqrt{\frac{P_{i n} R_{R}^{r a d}}{2}} G_{R} R_{n}^{r a d} G_{n}\left(\underline{\hat{h}}_{n} \cdot \underline{\hat{h}}_{R}\right)^{2} \frac{e^{-j 2 k r_{n}}}{4 r_{n}^{2}}
$$

where $\lambda$ is the wavelength, $P_{i n}$ is the power supplied by the reader, $R_{R}^{r a d}$ and $R_{n}^{r a d}[d]$ are, respectively, the radiation resistance of the reader and of the $n$th tag antenna, $G_{R}$ is the gain of the reader antenna, $G_{n}[d]$ the gain of the $n$th tag (when the other tag is in open circuit condition), $\underline{\hat{h}}_{R}$ and $\underline{\hat{h}}_{n}[d]$ are respectively the polarization unitary vector of the reader and of the $n$th tag antenna. Since the displacements of interest (millimeter and sub-millimeter scale) are much smaller than the reader-to-tag distance, it is here and in the following assumed that the effective heights of the couplet will undergo negligible variations with mutual distance.

The phase of the signal backscattered from the $n$th modulating tag and retrieved by a commercial reader can be extracted (as for instance reported in [13]) by the differential received signal among the two modulating impedance states

$$
\varphi_{n}[d]=\arg \left\{V_{R,(n)}^{O F F}[d]-V_{R,(n)}^{O N}[d]\right\} .
$$

Introducing (1) in (3) and denoting with $Z_{S}=Z_{11}=Z_{22}$ (self impedance) and $Z_{M}=Z_{21}=Z_{12}$ (mutual impedance), and considering a reference modulation scheme [14] having $Z_{C, n}^{O N}=$ $Z_{C, m}=Z_{C}$ and $Z_{C, n}^{O F F} \rightarrow \infty$; hence, (3) becomes

$\varphi_{n}[d]=\arg \left\{-\frac{Z_{S}[d]-Z_{M}[d]+Z_{C}}{\left(Z_{S}[d]+Z_{C}\right)\left(Z_{S}[d]+Z_{M}[d]+Z_{C}\right)}\right\}+\varphi_{0}$

where the baseline phase $\varphi_{0}=\arg \left\{f\left(r_{n}\right)\right\}$ is independent from the impedances and will not change if the relative distance between the reader and the tag remains stable in successive measurements (fixed measurement setup assumption), under the simplifying hypothesis previously made.

The unknown displacement $\delta$ between A, B points, and, hence, the evolution of the crack, can be hence formally estimated by inverting the calibration curve obtained as phase difference at a given time with respect to the initial reference condition

$$
\delta \leftrightarrow \Delta \varphi_{n}=\varphi_{n}[d]-\varphi_{n}\left[d_{0}\right] .
$$

\section{Antenna Design Problem}

Equation (5) gives a sensing metric to be considered in the application of the two-tags system whose performance can be quantified in terms of sensitivity $S=\partial \varphi / \partial \delta$. Anyway, since it has been demonstrated [11] that the features of a tag-as-a-sensor are generally obtained at the expenses of the degradation of the electromagnetic performance (the read distance), the design of the considered displacement sensor has also to cope with constraints over the active input impedance [12], [20] of the tags, which, if the reader illuminates the tags from boresight direction, can be written as

$$
Z_{i n, n}=Z_{S}+Z_{M} .
$$

The maximum power delivered to the chip, and, therefore, the maximum read distance, is obtained under Hermite matching condition $Z_{i n, n}=Z_{S}+Z_{M}=Z_{c h i p}^{*}$. The active input impedance can, hence, be assumed as the metric for the communication performance of the tag, since it is essential to guarantee maximum power delivered to the chip all along the sensing process.

It is worth noticing that being the self and mutual impedances dependent on the distance between the two tags, the active input impedance, and accordingly, the read distance of the two tags, will be sensitive to the displacement $\delta$. Thus, the goal of the antenna design is to achieve the maximum phase variation and the minimum change of the input impedance all along the evolution of the crack. An idea about how to master these constraints comes from a deeper understanding of the electromagnetic coupling of tags in case of very small inter-antenna distance.

\section{A. Extremely Coupled Antennas}

For two very close antennas, as in the case of the proposed two-tags displacement sensor, the coupled-modes theory [15] shows the onset of two families of current distributions, denoted as even and odd modes, according to the directions assumed by the induced currents over the antennas. The excitation of one or both the modes depends on the feeding modality, including the direction and polarization of the incoming field in case of receiving antennas. It is possible to define active input impedances for the even and odd modes as a function of the impedance matrix

$$
\begin{aligned}
& Z_{e}(d)=Z_{S}(d)+Z_{M}(d) \\
& Z_{o}(d)=Z_{S}(d)-Z_{M}(d) .
\end{aligned}
$$

More specifically, the two-tags RFID sensor, by the effect of the very close proximity, can be assumed to be excited by equalphase and equal-amplitude currents induced by the impinging field and, hence, the sensor will sustain only the even mode radiation.

As a matter of fact, the active input impedance of the 2-ports RFID grid, as defined in (6), corresponds to the input impedance (7) of the even-mode and, therefore, (4) and (6) can be rewritten (having omitted for notation simplicity the dependance on $d$ ) by introducing (7) and (8) as

$$
\varphi_{1}=\arg \left\{-\frac{\left(Z_{o}+Z_{C}\right)}{\left[\frac{1}{2}\left(Z_{e}+Z_{o}\right)+Z_{C}\right]\left(Z_{e}+Z_{C}\right)}\right\}+\varphi_{0}
$$

$$
Z_{i n, n}=Z_{e}
$$

As a consequence, the degradation of communication performance versus changes in the mutual distance of tags will be minimized if the even mode does not vary strongly along with the sensing process. The phase, instead, depends in a nonstraightforward manner on both even and odd-mode impedance and it is, hence, expected to exhibit substantial changes when either $Z_{e}$ or $Z_{o}$ have strong variations with mutual distance. In other 


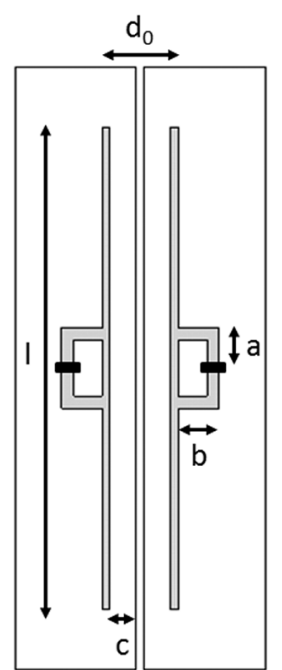

Fig. 2. Closely coupled T-match dipoles over a $150 \times 30 \times 0.8 \mathrm{~mm}$ FR-4 slab. Size in [mm]: $a=10, b=12, c=6.5, l=120$, while $d$ is considered variable.
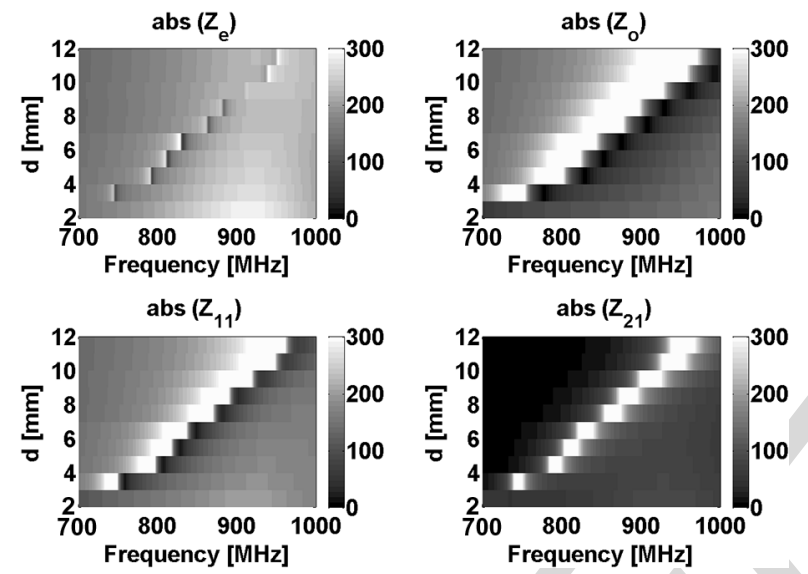

Fig. 3. Simulated even and odd mode (top) and self and mutual (bottom) impedances (absolute values) versus frequency and displacement for the two strongly-coupled T-match dipoles of Fig. 2.

words, conditions have to be found such that the even mode impedance is stable over inter-tag distance evolution, while the odd mode changes substantially.

The above concepts can be made explicit by the help of an example concerning two closely coupled T-match dipoles (Fig. 2) laying onto an FR-4 substrate.

Fig. 3 shows the absolute value of self, mutual, even and odd impedances with respect to inter-antenna distance and frequency, as obtained by numerical simulation through the electromagnetic solver CST Microwave Studio [18]. It is possible to clearly observe that self, mutual and odd impedances experience strong variations over frequency and over inter-antenna distance, while the even mode impedance is considerably more stable.

On recalling that $Z_{e}=Z_{i n, n}$, the combinations of $\{d, f\}$ around the main diagonal of the diagrams in Fig. 3 are particularly interesting since the input impedance of the tags remains quite stable for various mutual distances, while at the same time self and mutual impedances, as well as the odd mode impedance, change rapidly.
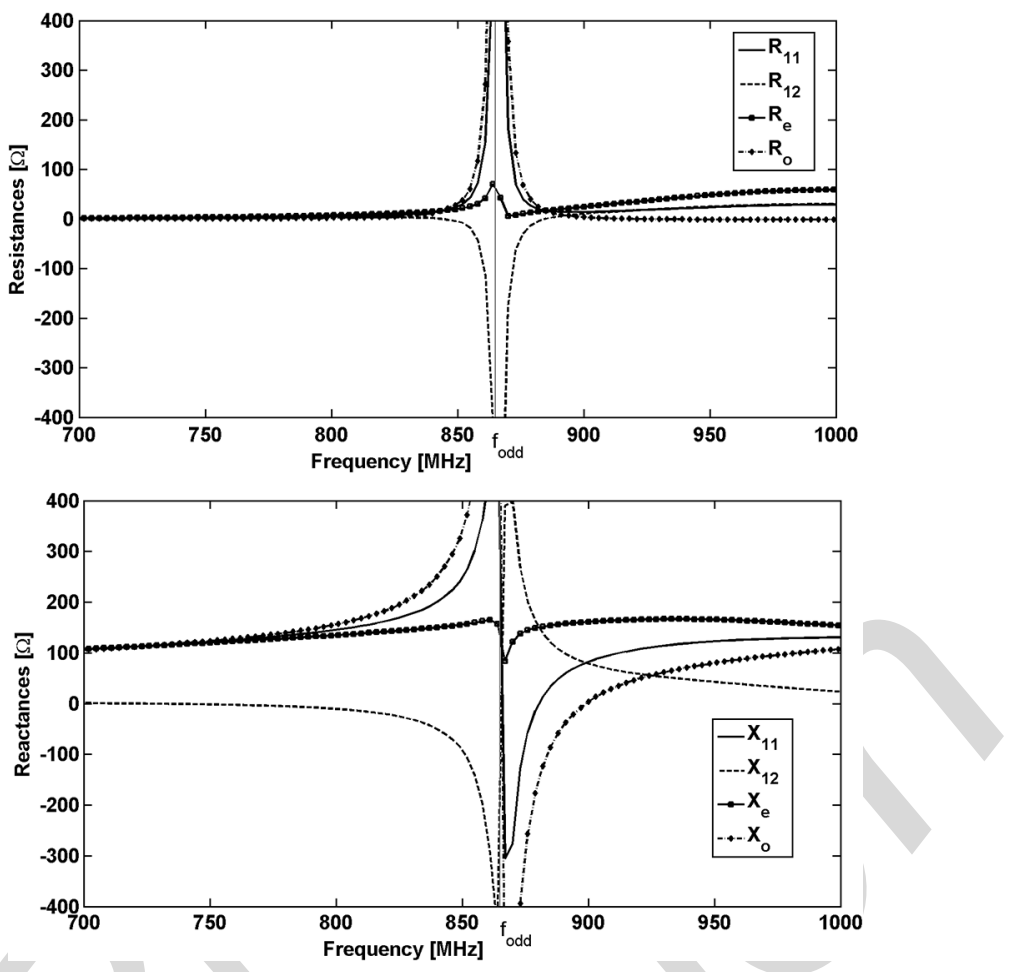

Fig. 4. Self and mutual resistance (top) and reactance (bottom) for the simulated couplet of the T-match tags of Fig. 2 at a mutual distance of $d_{0}=7 \mathrm{~mm}$ : the onset of the even and odd modes is clearly visible.

Forcing the tags to operate in the high-impedance band of Fig. 3 will, therefore, allow large phase variations with moderate degradation of impedance matching.

More in details, Fig. 4 shows a cut of previous impedance maps for a fixed inter-antenna distance $d=7 \mathrm{~mm}$ : the frequency region where the interesting phenomena occur corresponds to the parallel resonance of the odd mode input impedance and can be easily identified. These properties are of structural kind and hold also for different antenna layouts.

\section{B. Design Procedure}

The structural properties of the two closely coupled tags can be used to set up a design procedure that aims at forcing the two tags to operate in the high sensitive impedance region of Fig. 3. Let $\boldsymbol{\alpha}=\left\{\alpha_{1}, \alpha_{2}, . ., \alpha_{M}\right\}$ denote the set of geometrical and/or electrical parameters of the tags to be optimized, formally including also the initial inter-tag distance. However, for the sake of simplicity, and to speed-up the optimization procedure, a fixed reference distance $d=d_{0}$ is hereafter assumed. For instance, $d_{0}$ could be the smallest inter-tag distance that is compatible with the mechanical alignment of real antennas, including the substrates and the fixing method. The useful span of displacement detectable by the sensor will be verified a-posteriori at the end of the optimization procedure. As shown later on in the example section, the definitive initial inter-tag spacing for application on the crack will be then chosen according to the expected expanding or compressing behavior of the crack or, more in general, the initial distance could be allocated in the middle of the useful displacement range.

Parameters $\alpha$ have to be selected so that: 
i) the odd-mode anti-resonant frequency be tuned to the RFID frequency, i.e. $f_{r e s, O D D} \rightarrow f_{R F I D}$ to achieve enough sensitivity and negligible variation of the active input impedance and, hence, of the tags' range during the surface deformation process;

ii) the active input impedance be subjected to Hermite matching e.g. $Z_{i n, n}[d]=Z_{e}[d] \approx Z_{C}^{*}$ to enable the optimal delivery of scavenged power to the tags' microchip. The above constraints are included into a penalty function $P[\boldsymbol{\alpha}]$

$$
P[\boldsymbol{\alpha}]=w_{1} \frac{\left|Z_{i n, n}[\boldsymbol{\alpha}]-Z_{c}^{*}\right|}{\left|Z_{c}^{*}\right|}+w_{2} \frac{\left|f_{r e s, O D D}[\boldsymbol{\alpha}]-f_{R F I D}\right|}{\left|f_{R F I D}\right|}
$$

to be minimized by means of any optimization tool. The weighting coefficients $w_{1}$ and $w_{2}=1-w_{1}$ may be freely chosen to emphasize sensing or communication performance.

For each guess of antenna parameters $\boldsymbol{\alpha}^{(k)}$, the anti-resonance frequency $f_{r e s, O D D}\left[\boldsymbol{\alpha}^{(k)}\right]$ of the odd-mode impedance (8) is detected by a zero-seeking procedure and compared with the RFID frequency. The impedance mismatch is estimated from (6) for $d=d_{0}$ and compared with the ideal Hermite matching condition, hence contributing to the evaluation of the penalty function.

Once the optimal antenna parameters have been determined through optimization, a further numerical electromagnetic simulation is performed, varying the inter-antenna distance $d$ around the reference distance $d_{0}$, in order to calculate the spacing-dependent two-port impedance matrix and to accordingly estimate the variation of the phase and of the realized gain all along the displacement process. A design example is given in the next section.

\section{Numerical EXAMPLES}

The application of the above design procedure is now discussed in detail by means of the same T-Match dipoles layout as in Fig. 2. The tags are assumed to operate in the European UHF band 865.6-867.6 MHz and to be connected to RFID microchips having input impedance $Z_{c, 1}=Z_{c, 2}=Z_{c}=15-$ $j 135 \Omega$ at $f_{R F I D}=867 \mathrm{MHz}$ (resembling that of NXP G2XM chip). The geometrical parameters of the two-tags sensor that are available to the optimization procedure are the T-match's form factor $\{a, b\}$ and the dipole length $l$ so that $\boldsymbol{\alpha}=\{a, b, l\}$. In particular, $\{a, b\}$ are known to mostly affect the impedance matching while $l$ impacts on the resonant frequency. The reference inter-dipole distance is fixed to $d_{0}=7 \mathrm{~mm}$ and the tag responses will be evaluated for $|\delta| \leq 5 \mathrm{~mm}$. In these conditions, the two tags are partially overlapping because of the dimensions of the tags shown in Fig. 2 and one tag slides over the other.

Fig. 5 shows the penalty function computed as described above with respect to parameters $b / a$ and $l$ and for the choice of the weights $w_{1}=0.3, w_{2}=0.7$, i.e. giving slightly more attention to the device sensitivity than to the communication capability. The penalty function gets minimized in the surrounding of $\{l, a, b\}=\{120,10,12\} \mathrm{mm}$. In this condition, we obtain $Z_{\text {in }}\left(f_{\text {RFID }}\right)=8+j 145 \Omega$ (to be compared with the conjugate of the microchip impedance) and $f_{r e s, O D D}=862 \mathrm{MHz}$ (to be compared with the European UHF RFID frequency).

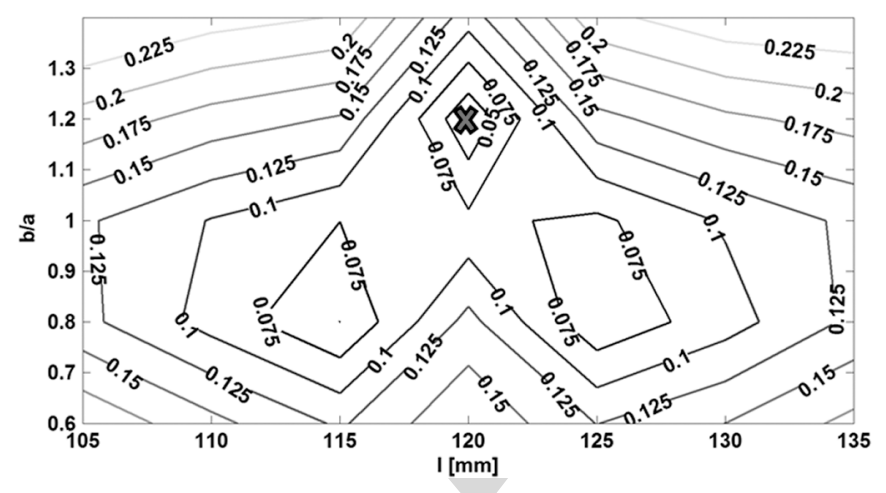

Fig. 5. Contours of the penalty function $P[l, b / a]$ for the two-dipoles sensor at $f_{R F I D}=867 \mathrm{MH}$. (having chosen $w_{1}=0.3, w_{2}=0.7$ ), with respect to the dipole length and the T-match aspect ratio. The optimal solution is marked by a "X".

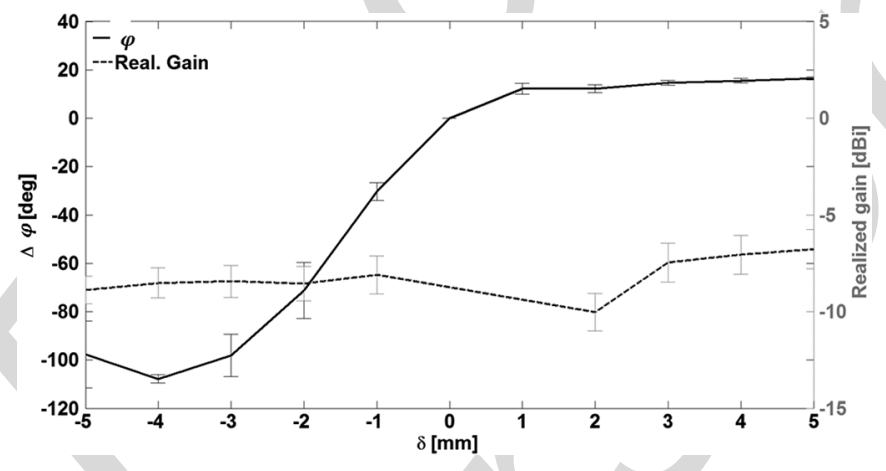

Fig. 6. Simulated phase calibration-curve (straight line) at $f=867 \mathrm{MH} z$, and realized gain (dotted line) versus the displacement $\delta$ among dipoles with respect to the reference configuration $d_{0}=7 \mathrm{~mm}$, so that $\overline{A B}=d_{0}+\delta$. Error bars indicate the sensitivity of the tag's response to $\pm 10 \%$ uncertainty in the microchip impedance w.r.t. the nominal value.

Finally, the calibration curve relating the relative phase $\triangle \varphi(\delta)$ computed as in (5) is represented in Fig. 6 together with the realized gain of the two tags-sensor, that can be considered an indirect measure of the impedance matching of the tags, easily retrieved by measurement throughout the turn-on method, as shown later in the experimental section. ${ }^{1}$

A monotonic trend is visible for the phase versus the intertags displacement with a nearly linear profile for $-4 \mathrm{~mm} \leq$ $\delta \leq 1 \mathrm{~mm}$ for an overall phase dynamic range of $120^{\circ}$. The estimated sensitivity is $S(\delta)=\partial \varphi / \partial \delta \sim 20[\mathrm{deg} / \mathrm{mm}]$. The realized gain is instead quite constant throughout the useful displacement range and is such to provide a maximum read distance of $3 \mathrm{~m}$ (for $E I R P=3.2 \mathrm{~W}$ ) in free space condition.

The error bars in the sensor's response are related to a possible $\pm 10 \%$ uncertainty over the true input impedance of the RFID chip which is subjected to changes with respect to the nominal value provided by the data-sheet, depending on the fixing modality and on the incident power level. Although not negligible, such uncertainty does not invalidate the overall behavior of both phase and realized gain versus displacement and can afterwards be removed through calibration of the sensor.

\footnotetext{
${ }^{1}$ The realized gain of the two tags' sensor, which is directly proportional to the read distance, is defined [12] as $\widetilde{G_{n}}=G_{n}\left(4 R_{C} R_{S}^{r a d} /\left|Z_{i n, n}+Z_{C}\right|^{2}\right) \chi_{n}$, where $\chi_{n}$ is the polarization mismatch between reader and tag, $R_{C}=\operatorname{Re}\left[Z_{c}\right]$ and $R_{S}^{r_{a d}}$ is the radiation resistance of each tag antenna in case the other is in open circuit condition.
} 


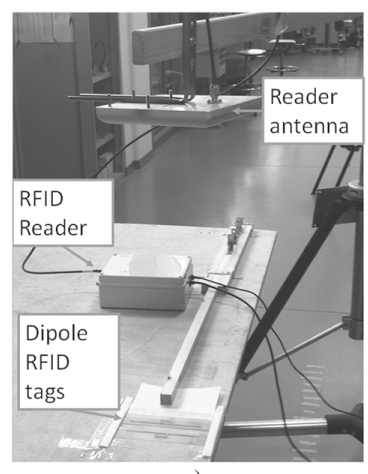

a)

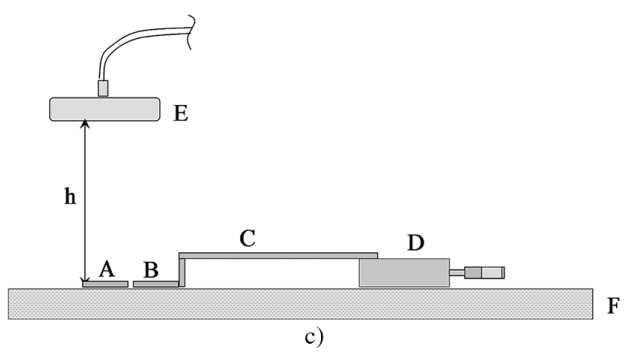

Fig. 7. a) Measurement setup, with two T-match tags over FR-4 placed on a wooden desk in front of the reader's antenna: the sliding tag translates by means of the linear translation stage guide. b) Zoom on the manufactured T-match tags. c) Detailed scheme of the set-up: A fixed tag; B sliding tag; C wooden rod; D linear translation stage guide; E reader's antenna; F wooden desk.

\section{EXPERIMENTATION}

The T-match dipole couplet of the previous Section has been manufactured using a drilling and milling machine for PCB prototyping. Antennas and T-match are carved out of a single-face FR-4 board $0.8 \mathrm{~mm}$ thick; NXP-G2XM microchips are soldered at the antenna terminals [Fig. 7(b)]. The two tags are closely deployed on a wooden desk so that one of the tags is fixed on the desk (fixed tag) while the other (sliding tag) shifts orthogonally (to the longer side) by means of a linear translation stage guide with resolution $\Delta \delta=10^{-2} \mathrm{~mm}$ [Fig. 7(a)]. The reader's antenna, a circularly polarized patch with $6 \mathrm{dBi}$ gain, is standing above the tags at distance $h=0.7 \mathrm{~m}$ and is connected to a ThingMagic M6e reader [19] set for fixed frequency operation and having disabled the frequency hopping. The reference inter-tag distance was fixed to $d_{0}=7 \mathrm{~mm}$, as in the optimization task. The sliding tag was progressively moved in the range $d_{0}-5 \mathrm{~mm}<d<d_{0}+5 \mathrm{~mm}$ by steps of 0.5 $\mathrm{mm}$ for an overall displacement of $10 \mathrm{~mm}$. RFID interrogations are performed at each steps: measurements consist in collecting the phase of the received backscattered signals and the turn-on power, which will be used to calculate the realized gain, as in [17].

Two measurement sessions have been performed for co-axial orientation between reader and tags (boresight measurement) and for off the axis placement (off-boresight measurement) with the aim to investigate the impact of imperfect alignments.

\section{A. Measurement Along the Boresight}

In a first experiment, the reader's boresight is aligned to the two-tags' normal axis so that the hypothesis in Section II are fully matched. The variation of the phase of the two tags recorded at the reader versus the distance between the two tags

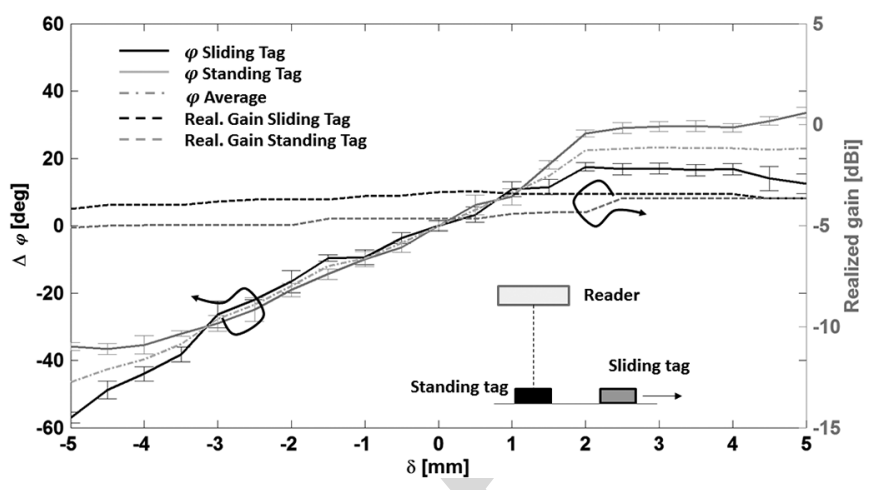

Fig. 8. Measured phase variation and realized gain (at $867 \mathrm{MHz}$ ), for the fixed and sliding tags, versus displacement $\delta$ among tags with respect to reference inter antenna distance $d_{0}=7 \mathrm{~mm}$ in case of along boresight illumination. Bars indicate the standard deviation in repeated measurements.

with increasing steps of $\Delta \delta=0.5 \mathrm{~mm}$ is shown in Fig. 8, together with the corresponding realized gain. Vertical bars in the diagram indicate the standard deviation in repeated measurements which resulted to be less than $3^{\circ}$. The useful part of the dynamic range of the sensor, wherein the profile is mostly linear, is $-4 \mathrm{~mm} \leq \delta \leq 2 \mathrm{~mm}$. It is worth specifying that, having assumed an arbitrarily reference initial inter-antenna spacing $d_{0}=7 \mathrm{~mm}$, the absolute spacing that can be sensed by the systems will be $3 \mathrm{~mm}<d=d_{0}+\delta<9 \mathrm{~mm}$. Hence, if, for instance, an expanding crack is expected, the full available $6 \mathrm{~mm}$ sensing range can be exploited by fixing the two antenna at an initial distance $d^{\prime}=3 \mathrm{~mm}$. The experimented sensitivity is $S_{\text {meas }}(\delta) \sim 10 \mathrm{deg} / \mathrm{mm}$ in the range $\delta=\{-4 ; 2\} \mathrm{mm}$ to be compared with the theoretical value $S_{\text {theor }}(\delta) \sim 20 \mathrm{deg} / \mathrm{mm}$. Differences are mostly due to the presence of the wooden desk set-up, on which tags are placed and that was instead not considered in the optimization.

Finally, it is worth noticing that the phase profiles of the sliding and standing tags are quite similar in the useful linear region, while some asymmetries are apparent elsewhere, maybe due to the partial overlapping of the FR-4 supports of the two tags and to the potential difference between the two microchip RF impedances, as discussed in Fig. 6. It is, hence, suggested to perform a calibration task (numerical and/or experimental), at least for the specific class of applications, to get rid of possible material-dependent uncertainty, before real-life use.

\section{B. Off Boresight Measurement}

A second set of experiments (Fig. 10) considers shifts and rotations (Fig. 9) of the reader's antenna w.r.t. the boresight alignment, so that the two-tags sensor is illuminated from different positions $\{\theta, \phi, r\}$. These experiments permit to evaluate the immunity of the sensor's response from the particular choice of mutual reader-tag alignment (that, however, is considered fixed during each measurement session of the inter-tag displacement).

Fig. 10 shows the results for a moderate deviation from the boresight illumination and for $10 \mathrm{~cm}$ increase in the reader-tag distance $\left(\{\theta, \phi, r\}=\left\{25^{\circ}, 90^{\circ}, 80 \mathrm{~cm}\right\}\right)$. The obtained displacement-phase profile fully compares with that of the reference boresight case. 


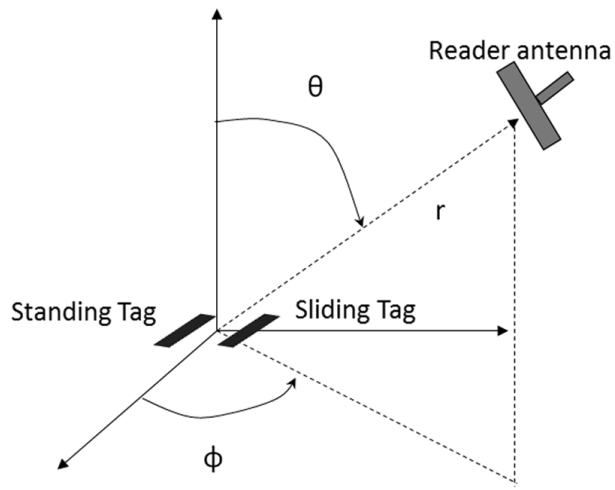

Fig. 9. Geometrical setup used to test the sensor's response for different readertags positions.

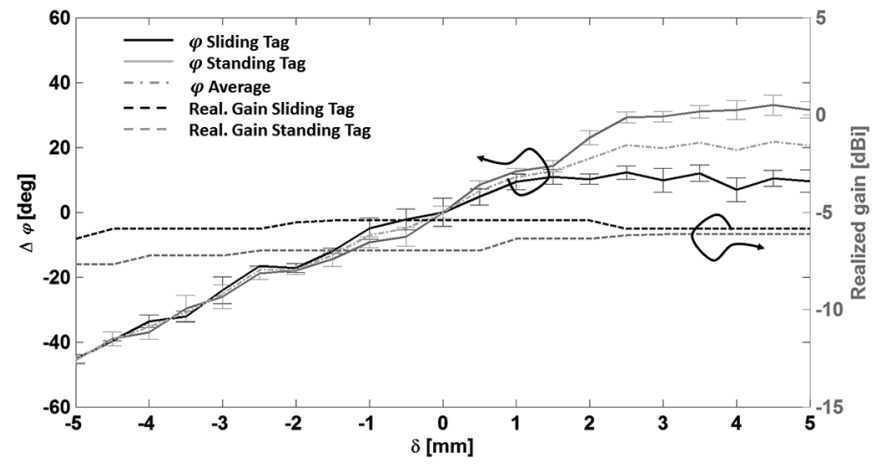

Fig. 10. Measured phase variation and realized gain (at $867 \mathrm{MHz}$ ), for the fixed and sliding tags, versus displacement $\delta$ among tags with respect to reference inter-antenna distance $d_{0}=7 \mathrm{~mm}$ in case of off-boresight illumination $\theta, \phi, r=\left\{25^{\circ}, 90^{\circ}, 80 \mathrm{~cm}\right\}$.

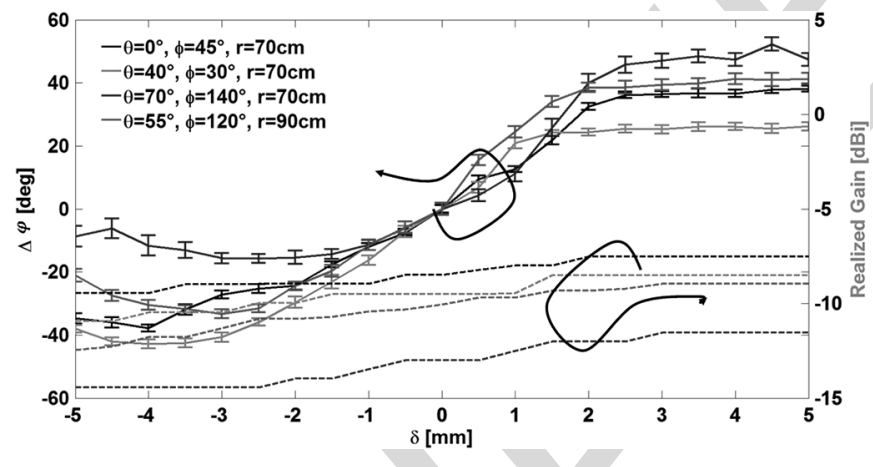

Fig. 11. Measured phase variation and realized gain (at $867 \mathrm{MHz}$ ) versus displacement $\delta$ among tags with respect to reference inter-antenna distance $d_{0}=$ $7 \mathrm{~mm}$ in case of several off-boresight illumination.

Results for more severe grazing illuminations $\left(40^{\circ}<\theta<\right.$ $70^{\circ}$ ) are summarized in Fig. 11 for the fixed tag, at a distance up to $r=90 \mathrm{~cm}$ from the reader.

On comparing the response profiles to the one obtained for boresight illumination, it is possible to appreciate a substantial stability in the central part of the linear region (around $-3 \mathrm{~mm}<\delta<2 \mathrm{~mm}$ ) except for the very low elevation angles $\left(\theta=70^{\circ}\right)$, where the simplifying hypotheses of Section II are not fulfilled anymore.

It is worth concluding that, while the sensor's gain is quite strongly affected by the mutual orientation between devices, the reader-tag mutual position will not be instead a critical issue in

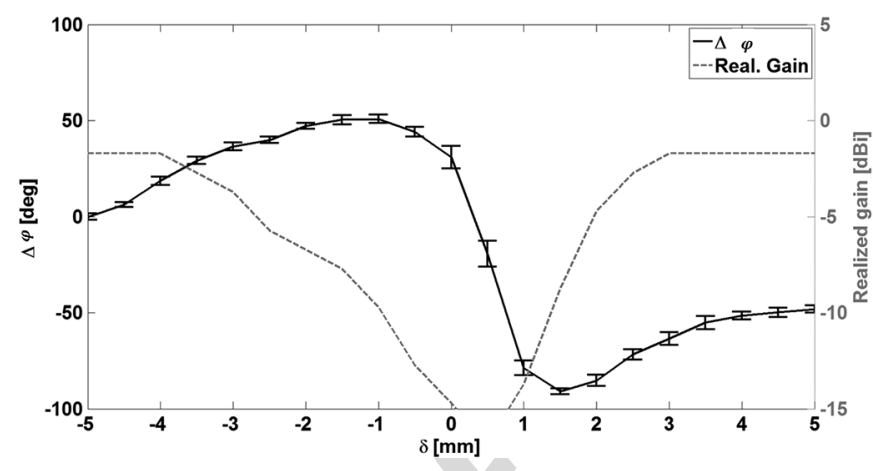

Fig. 12. Measured phase variation and realized gain (at $867 \mathrm{MHz}$ ) versus displacement $\delta$ among tags with respect to reference inter-antenna distance $d_{0}=$ $7 \mathrm{~mm}$ in case of only one tag with chip and the other in OC; boresight illumination.

real life phase measurements, provided that the elevation angles does not roughly exceed $45^{\circ}$. It is, however, advisable to perform calibration with the same (fixed) setup used in measurement to reduce uncertainties related to the random mutual orientation.

\section{A Single-Chip Case}

It is finally useful to analyze the sensing performance achievable when only a single chip is used, e.g. when the chip of the sliding antenna is replaced by an open circuit. The resulting device will, hence, work as a moving disturbing scatterer for the true RFID tag, as in the case of the sliding patch in [10]. The balancing effect of the mutual interaction between the tags will be lost, since, if $Z_{C, 2} \rightarrow \infty$, phase and active input impedance will approach the simplified expressions

$$
\begin{aligned}
\varphi_{1} & \rightarrow \arg \left(\left(\frac{1}{Z_{S}+Z_{C, 1}}\right)\right)+\varphi_{0} \\
Z_{i n, 1} & \rightarrow Z_{s} .
\end{aligned}
$$

Since the self impedance exhibits strong variations at $f_{R F I D}$ for inter-tag displacements around $d=7 \mathrm{~mm}$ (as in Fig. 4), both phase and input impedance are expected to vary strongly.

Fig. 12 shows the measured results from laboratory experiments $\left(\theta, \phi, r=\left\{0^{\circ}, 0^{\circ}, 100 \mathrm{~cm}\right\}\right)$ : a much higher sensitivity $S(\delta)=150 \mathrm{deg} / \mathrm{mm}$ is achieved but within a more limited range of displacement $\delta=[0 ; 1] \mathrm{mm}$, and at the expense of a stronger degradation in the link range, since the realized gain $\widetilde{G_{n}}$ attenuates by more than $10 \mathrm{~dB}$.

\section{CONCLUSION}

Relating the phase of the backscattering power emitted by two strongly coupled facing tags to the change of their mutual position has been demonstrated to be a promising procedure to detect sub-millimeter displacements without producing strong communication degradation that is instead typical of amplitude-oriented RFID sensors. On considering that a commercial low-cost reader typically exhibits a phase resolution of $3^{\circ}$, the results of experimentation show that the proposed couplet of tags already allows a displacement resolution as low as $0.3 \mathrm{~mm}$ over a span of up to $6 \mathrm{~mm}$. That resolution could be improved by further optimization and trade-off analysis (i.e. by giving more 
emphasis to sensitivity rather than communication in the design procedure), as well as by using more stable readers with great benefit to spatial resolution. For instance, a phase deviation of $1^{\circ}$ could enable a displacement resolution of $0.1 \mathrm{~mm}$ with the proof-of-concept manufactured prototype: such value comes near the one of some well-assessed active measurement devices. In case very short range interrogations are acceptable, even sharper resolutions could be moreover achieved by using single-chip configurations where the dummy tag works as a sliding scatterer.

The proposed technique employing phase to retrieve sensing information needs a fixed measurement setup, even if it revealed rather robust to the imperfect alignment between reader's antenna and tags. The stationary-measurement requirement, that looks not suitable to the use of hand-held readers, is instead fully compliant with laboratory environments as well as with external real life applications provided that at least the reader's antenna (and not necessarily the whole reader unit) remains fixed with respect to the surface to be observed at different times. In this sense, the proposed arrangement could be particular feasible in avionic framework wherein the interrogating antenna could be integrated inside the structure.

\section{REFERENCES}

[1] C. Boller and N. Meyendorf, "State of the art in SHM for aeronautics," presented at the Int. Symp. NDT Aerospace, 2008.

[2] P. C. Chang, A. Flatau, and S. C. Liu, "Review paper: Health monitoring of civil infrastructure," Struct. Health Monit., vol. 2, no. 3, pp. 257-267, Sep. 2003.

[3] C. Occhiuzzi, S. Caizzone, and G. Marrocco, "Passive UHF RFID antennas for sensing applications: Principles, methods and classifications," IEEE Antennas Propagat. Mag., to be published.

[4] K. Morita and K. Noguchi, "Crack detection methods for concrete and steel using radio frequency identification and electrically conductive materials and its applications," Proc. SPIE, 2008.

[5] C. Occhiuzzi, C. Paggi, and G. Marrocco, "Passive RFID strain-sensor based on meander line antennas," IEEE Trans. Antennas Propag., vol. 59, no. 12, pp. 4836-4840, Dec. 2011.

[6] B. S. Cook, A. Shamim, and M. M. Tentzeris, "Passive low-cost inkjetprinted smart skin sensor for structural health monitoring," IET Microw. Antennas Propag., vol. 6, no. 14, pp. 1536-1541, Nov. 2012.

[7] P. Kalansuriya, R. Bhattacharyya, S. Sarma, and N. Karmakar, "Towards chipless RFID-based sensing for pervasive surface crack detection," in Proc. IEEE Conf. RFID- Technologies and Applications, Nov. 2012, pp. 46-51.

[8] X. Yi, C. Cho, C.-H. Fang, J. Cooper, V. Lakafosis, R. Vyas, Y. Wang, R. T. Leon, and M. M. Tentzeris, "Wireless strain and crack sensing using a folded patch antenna," in Proc. Eur. Conf. Antennas and Propagation, Mar. 2012, pp. 1678-1681.

[9] V. Rizzoli, A. Costanzo, E. Montanari, and A. Benedetti, "A new wireless displacement sensor based on reverse design of microwave and millimeter-wave antenna array," IEEE Sensors J., vol. 9, no. 11, pp. 1557-1566, Nov. 2009.

[10] C. Paggi, C. Occhiuzzi, and G. Marrocco, "Sub-millimeter displacement sensing by passive UHF RFID antennas," IEEE Trans. Antennas Propag., vol. 62, no. 2, pp. 905-912, Feb. 2014.

[11] C. Occhiuzzi and G. Marrocco, "Constrained design of passive UHF RFID sensor antennas," IEEE Trans. Antennas Propag., vol. 61, no. 6, pp. 2972-2980, Jun. 2013.

[12] G. Marrocco, "RFID grids: Part I-Electromagnetic theory," IEEE Trans. Antennas Propag., vol. 59, no. 3, pp. 1019-1026, Mar. 2011.

[13] P. V. Nikitin, R. Martinez, S. Ramamurthy, H. Leland, G. Spiess, and K. V. S. Rao, "Phase based spatial identification of UHF RFID tags," in Proc. IEEE Int. Conf. RFID, Apr. 2010, pp. 102-109.

[14] F. Fuschini, C. Piersanti, F. Paolazzi, and G. Falciasecca, "Analytical approach to the backscattering from UHF-RFID transponder," IEEE Antennas Wireless Propag. Lett., vol. 7, pp. 33-35, Feb. 2008.
[15] H. A. Haus and W. Huang, "Coupled-mode theory," Proc. IEEE, vol. 79, no. 10, pp. 1505-1518, Oct. 1991.

[16] S. Manzari, S. Pettinari, and G. Marrocco, "Miniaturised wearable UHF-RFID tag with tuning capability,” Electron. Lett., vol. 48, no. 21, pp. 1325-1326, Oct. 2012.

[17] G. Marrocco and F. Amato, "Self-sensing passive RFID: From theory to tag design and experimentation," presented at the Eur. Microwave Conf., Sep. 2009.

[18] [Online]. Available: http://www.cst.com

[19] [Online]. Available: http://www.thingmagic.com/embedded-rfidreaders/mercury6e

[20] C. Balanis, Antenna Theory- Analysis and Design. Hoboken, NJ, USA: Wiley, p. 413.

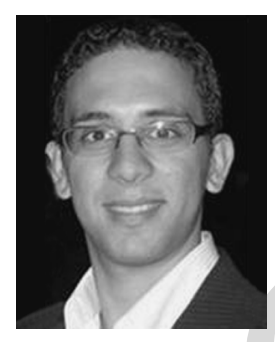

Stefano Caizzone received the M.Sc. degree in telecommunications engineering from the University of Rome Tor Vergata, Italy, in 2009. He is currently pursuing the Ph.D. degree.

His main research interests concern small antennas for RFIDs and navigation, antenna arrays, and grids with enhanced sensing capabilities. He is now with the Antenna Group, Institute of Communications and Navigation, German Aerospace Center (DLR), Wessling, Germany, where he is responsible for the development of innovative miniaturized antennas.

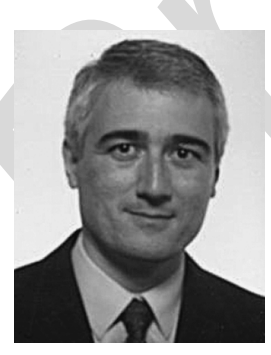

Emidio DiGiampaolo received the Laurea degree in electronic engineering and the Ph.D. degree in applied electromagnetics from the University of L'Aquila, Italy, in 1994 and 1998, respectively.

From 1998 to 2004, he was a postdoctoral researcher at the University of L'Aquila. In the spring of 2000, he was a visiting researcher at the European Space Research and Technology Centre (ESTEC), Noordwijk, The Netherlands. From 2005 to 2009, he was a researcher at the University of Rome Tor Vergata, Italy. Since 2010, he has been with the University of L'Aquila as an Assistant Professor. His research interests mainly concern numerical methods for modeling radio-wave propagation in complex environments, antennas, RFID, and radio localization.

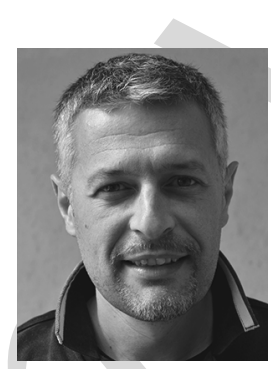

Gaetano Marrocco received the electronic engineering Laurea and the Ph.D. degree in applied electromagnetics from the University of L'Aquila, Italy, in 1994 and 1998, respectively.

In 1994, he was with the University of Illinois at Urbana-Champain as a postgraduate student. In 1997, he joined the University of Roma Tor Vergata, Italy, as a researcher. In 1999, he was a visiting researcher at the Imperial College, London, U.K. Currently, he serves as Associate Professor of electromagnetics, University of Roma Tor Vergata, and he chairs the Pervasive Electromagnetics Lab. In 2014, he obtained the Full Professor qualification. In 2015, he will be a Guest Professor at the University of Paris-est Marne la Vallèe, France. His research is mainly focused on the application of radiofrequency identification (RFID) to medical and industrial diagnostics as well as to the modeling and design of distributed and miniaturized conformal antenna clusters over ships, micro and nano-satellites, and aircrafts within the framework of the European Space Agency, NATO, the Italian Space Agency, and the Italian Navy research projects. Moreover, he was active in the development of FDTD methods for microwave hyperthermia and in the modeling and design of pulsed arrays.

Prof. Marrocco serves as an Associate Editor of the IEEE ANTENNAS AND Wireless PROPAGATION LETTERS and the IEEE RFID VIRTUAL JOURNAL and as Vice-Chair of the Italian delegation URSI Commission D: Electronics and Photonics. He was the Chair of Local Committee of EUCAP-2011, Rome, Italy, and TPC Chair of the 2012 IEEE-RFID TA, Nice, France. He is the cofounder and President of the University spin-off RADIO6ENSE that is active in the short-range electromagnetic sensing for industry, internet of things, and smart cities. 